\title{
One-loop matching in the SMEFT extended with a sterile neutrino
}

\author{
Mikael Chala ${ }^{a}$ and Arsenii Titov ${ }^{b}$ \\ ${ }^{a}$ CAFPE and Departamento de Fúsica Teórica y del Cosmos, Universidad de Granada, \\ E-18071 Granada, Spain \\ ${ }^{b}$ Dipartimento di Fisica e Astronomia "G. Galilei", Università degli Studi di Padova and \\ INFN, Sezione di Padova, \\ Via Francesco Marzolo 8, I-35131 Padova, Italy \\ E-mail: mikael.chala@cern.ch, arsenii.titov@pd.infn.it
}

ABSTRACT: We study the phenomenology of the simplest renormalisable model that, at low energy, leads to the effective field theory of the Standard Model extended with right-handed neutrinos ( $\nu$ SMEFT). Our aim is twofold. First, to contextualise new collider signatures in models with sterile neutrinos so far studied only using the bottom-up approach. And second and more important, to provide a thorough example of one-loop matching in the diagrammatic approach, of which other matching techniques and automatic tools can benefit for cross-checks. As byproducts of this work, we provide for the first time: (i) a complete off-shell basis for the $\nu$ SMEFT and explicit relations between operators linked by equations of motion; (ii) a complete basis for the low-energy effective field theory ( $\nu$ LEFT) and the tree-level matching onto the $\nu$ SMEFT; (iii) partial one-loop anomalous dimensions in the $\nu$ LEFT. This way, our work comprises a new step forward towards the systematisation of one-loop computations in effective field theories, especially if the SM neutrinos are Dirac.

Keywords: Beyond Standard Model, Effective Field Theories, Neutrino Physics

ARXIV EPRINT: 2001.07732 


\section{Contents}

1 Introduction 1

2 Model and effective description $\quad 3$

$\begin{array}{llr}3 & \text { Matching } & 6\end{array}$

4 Sterile neutrino phenomenology 11

5 Conclusions 13

A Mathematical tools $\quad 14$

B Details of computation of the UV amplitudes $\quad \mathbf{1 5}$

$\begin{array}{ll}\text { B.1 Amplitude for one } B \text { and no Higgs bosons } & 15\end{array}$

$\begin{array}{ll}\text { B.2 Amplitude for one Higgs and no gauge bosons } & 16\end{array}$

$\begin{array}{ll}\text { B.3 Amplitude for one Higgs and one photon } & 16\end{array}$

$\begin{array}{ll}\text { B.4 Amplitude for one Higgs and one } W & 17\end{array}$

$\begin{array}{lll}\text { B.5 Amplitude for two Higgses and no gauge bosons } & 18\end{array}$

$\begin{array}{ll}\text { B.6 } & \text { Amplitude for two Higgses and one } W^{3} \\ \end{array}$

$\begin{array}{ll}\text { B.7 Amplitude for three Higgses and no gauge bosons } & 19\end{array}$

$\begin{array}{ll}\text { B.8 Amplitude for four } N \text { fermions } & 19\end{array}$

B.9 Amplitude for two $N$ fermions and two neutrinos 20

$\begin{array}{ll}\text { C Matching of renormalisable terms } & 20\end{array}$

D Matching the $\nu$ SMEFT onto the $\nu$ LEFT and anomalous dimensions $\quad 24$

\section{Introduction}

Effective field theories (EFTs) are being used to describe the effects of new heavy particles at low energy in terms of operators of dimension higher than four. A well acknowledged advantage of this approach is its generality. The only model dependence resides in the light degrees of freedom out of which the EFT is built. (The symmetries of the EFT are in principle also debatable, but by now the group of gauge symmetries $\mathrm{SU}(3)_{c} \times \mathrm{SU}(2)_{L} \times \mathrm{U}(1)_{Y}$ is well established.) Among other aspects, this choice depends crucially on the nature of neutrinos. If neutrinos are Majorana, the simplest assumption is that the infrared (IR) comprises only the Standard Model (SM) fields. The resulting EFT, known as SMEFT [1, 2], has been extensively studied in the recent years; see ref. [3] for a fresh review. If neutrinos are Dirac, the low-energy sector has to be extended with right-handed $(\mathrm{RH})$ singlet 
fermions. The corresponding EFT is referred to as $\nu$ SMEFT $[4,5]$. It has also been applied to the case in which the new RH neutrinos are themselves Majorana, as predicted in numerous models. (See ref. [6] for an EFT for non-relativistic Majorana neutrinos.) With the same spirit, other EFTs have considered also new scalars in the IR; see e.g. refs. [7-9].

A common feature of all these EFTs is that they predict new processes that are completely absent in the renormalisable SM. Many of these processes have not been studied yet experimentally. These include, among others, rare decays of the top quark such as $t \rightarrow \ell^{+} \ell^{-} j[10,11], t \rightarrow b \bar{b} j[11,12]$ or the non-resonant $t \rightarrow b \ell^{+}+E_{T}^{\text {miss }}$ [13] as well as rare decays of the Higgs boson including $h \rightarrow \ell^{+} \ell^{-}+4 j$ [14], $h \rightarrow \gamma(\gamma)+E_{T}^{\text {miss }}$ [15]. (In ref. [16] the constraints on the $\nu$ SMEFT operators arising from low-energy experiments have been derived.) However, this bottom-up approach is not without drawbacks. Most importantly, operators other than those triggering the signals of interest are generally also present (with correlated coefficients) in concrete ultraviolet (UV) models; some of them being very constrained. Likewise, it is hard to prioritise one search over others.

It is therefore desirable that searches motivated by pure EFT inspection are also supported by realistic UV models. ${ }^{1}$ This exercise requires matching UV models to the EFT, generally at one loop (at which several of the most interesting and/or dangerous operators appear often). In the usual diagrammatic approach, this process consists of computing tens of one-light-particle-irreducible off-shell amplitudes in both the UV and the EFT. This is a very demanding task that in turn requires knowledge of a full off-shell basis of EFT operators (only those linked by algebraic identities and integration by parts being removed) and their relations by equations of motion. If low energy ( $E \ll v$, with $v \sim 246 \mathrm{GeV}$ being the Higgs vacuum expectation value) observables are to be computed, then the corresponding EFT in the electroweak (EW) symmetry broken phase must be also known, as well as its matching to the aforementioned operators. Renormalisation group evolution (RGE) of the Wilson coefficients in both EFTs might be also needed.

While several of these points have been already addressed in the SMEFT, ${ }^{2}$ very little is known about the $\nu$ SMEFT beyond a full (on-shell) basis of up to dimension-seven operators [4, 5, 23, 24]. Moreover, while new techniques [25-30] and tools [31-34] for one-loop matching are also being developed, a severe obstacle for progress in this respect is precisely the lack of explicit one-loop matching computations to which compare to in the literature [34]. (To the best of our knowledge, partial examples of one-loop matching have been only provided for the SM extended with a real scalar singlet [30, 35, 36], with a charged scalar singlet [37], with some colourless EW multiplets for very particular parameters [25] and with a vector-like quark singlet [31].)

\footnotetext{
${ }^{1}$ We are well aware that "realistic" is an arguable concept. Here we adopt the notion that a "realistic" UV model should involve less free parameters than the EFT (which in turn restricts the number of new independent heavy fields), and that there should not be large cancellations between different couplings of similar size.

${ }^{2}$ The first complete set of dimension-six operators was obtained in ref. [1]. Several of them were shown to be related by equations of motion in ref. [2]. The corresponding EFT below the EW symmetry breaking (EWSB) scale, known as LEFT, was worked out in ref. [17]; the tree-level matching of the SMEFT onto the LEFT was also provided in the same article. This computation has been recently performed at one loop in ref. [18]. Finally, the RGE of the SMEFT and LEFT operators was presented in refs. [19-21] and ref. [22], respectively.
} 
In light of the discussion above, in this paper we consider a simple UV model whose EFT description is the $\nu$ SMEFT, for which we provide a full off-shell basis and relations between different operators by equations of motion; see section 2 . In section 3 we perform the actual one-loop matching using the diagrammatic approach. We provide mathematical tools used and details of loop computations in appendix A and in appendices B and C, respectively. In section 4 we study the phenomenology of the resulting EFT (with operators with correlated Wilson coefficients, as they depend on only a very small number of UV couplings), both in the Majorana and in the Dirac cases, and highlight the importance of performing new Higgs searches at the LHC. To this aim, we also rely on a full on-shell basis of the EFT below the EW scale and its matching onto the $\nu$ SMEFT, as well as on partial RGE, all of which we provide in appendix D.

\section{Model and effective description}

We consider the SM extended with a light $\mathrm{RH}$ fermionic singlet $N$, as well as two heavy vector-like fermions $X_{E} \sim(\mathbf{1}, \mathbf{2})_{1 / 2}, X_{N} \sim(\mathbf{1}, \mathbf{1})_{1}$ and a heavy singly-charged scalar $\varphi \sim(\mathbf{1}, \mathbf{1})_{-1}$. The numbers within parentheses and the subindex indicate the representations of $\left(\mathrm{SU}(3)_{c}, \mathrm{SU}(2)_{L}\right)$ and the hypercharge $Y$, respectively. Relatively heavy vector-like fermions and/or charged scalars and (one or more) sterile neutrinos are present in a number of models motivated either phenomenologically (e.g. by the persistent discrepancy between the measured value of the muon anomalous magnetic moment and the corresponding SM prediction [38-40]) or theoretically (e.g. in models assuming left-right symmetry [41-43], grand unification [44] or compositeness $[45,46]$ - in this latter case vector-like fermions are strictly required by the partial compositeness paradigm [47].)

We assume $\mathrm{CP}$ and baryon number, while lepton number and lepton flavour conservation are only broken by the small (potentially vanishing) $N$ mass; $N$ is assumed to couple only to the electron (or to the muon; this choice does not alter our phenomenological results). Moreover, we assume that the heavy fields are odd under a $\mathbb{Z}_{2}$ symmetry under which all SM fields as well as $N$ are even.

We denote by $e, u, d$ the $\mathrm{RH}$ leptons and quarks; and by $L, Q$ the left-handed counterparts. We name the gluon and the EW gauge bosons by $G$ and $W, B$, respectively. Let us call the Higgs doublet by $H=\left[G^{+},\left(h+i G^{0}\right) / \sqrt{2}\right]$ and $\tilde{H}=i \sigma_{2} H^{*}$, with $\sigma_{I}, I=1,2,3$, being the Pauli matrices. The Lagrangian of this model reads:

$$
\mathcal{L}=\mathcal{L}_{\mathrm{SM}+N}+\mathcal{L}_{\text {heavy }},
$$

with

$$
\begin{aligned}
\mathcal{L}_{\mathrm{SM}+N}= & -\frac{1}{4} G_{\mu \nu}^{A} G^{A \mu \nu}-\frac{1}{4} W_{\mu \nu}^{I} W^{I \mu \nu}-\frac{1}{4} B_{\mu \nu} B^{\mu \nu} \\
& +\left(D_{\mu} H\right)^{\dagger}\left(D^{\mu} H\right)+\mu_{H}^{2} H^{\dagger} H-\frac{1}{2} \lambda_{H}\left(H^{\dagger} H\right)^{2} \\
& +i(\bar{Q} \not D Q+\bar{u} \not D u+\bar{d} \not D d+\bar{L} \not D L+\bar{e} \not D e+\bar{N} \not D N) \\
& -\left[\frac{1}{2} m_{N} \overline{N^{c}} N+\bar{Q} Y_{d} H d+\bar{Q} Y_{u} \tilde{H} u+\bar{L} Y_{e} H e+\bar{L} Y_{N} \tilde{H} N+\text { h.c. }\right],
\end{aligned}
$$


and

$$
\begin{aligned}
\mathcal{L}_{\text {heavy }}= & \overline{X_{E}}\left(i \not D-M_{X_{E}}\right) X_{E}+\overline{X_{N}}\left(i \not D-M_{X_{N}}\right) X_{N} \\
& +\left(D_{\mu} \varphi\right)^{*}\left(D^{\mu} \varphi\right)-M_{\varphi}^{2} \varphi^{*} \varphi-\lambda_{\varphi \varphi}\left(\varphi^{*} \varphi\right)^{2}-\lambda_{\varphi H}\left(\varphi^{*} \varphi\right)\left(H^{\dagger} H\right) \\
& +\left[g_{X} \overline{X_{E}} \tilde{H} X_{N}+g_{L} \overline{X_{E}} \varphi^{*} L+g_{N} \overline{X_{N}} \varphi^{*} N+\text { h.c. }\right] .
\end{aligned}
$$

Our conventions for the covariant derivative of a colour singlet field $\phi$ and for the EW field strength tensors are

$$
\begin{aligned}
& D_{\mu} \phi=\left(\partial_{\mu}-i g T^{I} W_{\mu}^{I}-i g^{\prime} Y B_{\mu}\right) \phi, \\
& W_{\mu \nu}^{I}=\partial_{\mu} W_{\nu}^{I}-\partial_{\nu} W_{\mu}^{I}+g \varepsilon^{I J K} W_{\mu}^{J} W_{\nu}^{K}, \quad B_{\mu \nu}=\partial_{\mu} B_{\nu}-\partial_{\nu} B_{\mu},
\end{aligned}
$$

where $T_{I}=\sigma_{I} / 2$ are the $\mathrm{SU}(2)$ generators.

This model features a number of interesting properties. (i) Because of the $\mathbb{Z}_{2}$ symmetry, if the heavy particles are integrated out, no effective operators arise at tree level. (Note also that this symmetry turns the neutral component of $X_{E}$ into a dark matter candidate, provided some mechanism at a higher scale - which does not modify the results below is invoked to avoid direct detection constraints; we do not elaborate on this aspect of the phenomenology though.) (ii) Because of this, it can be very easily shown that in the IR only tree-level amplitudes are to be computed while matching at one loop. ${ }^{3}$ (Actually, it can be shown that no loops need to be computed in the EFT even if tree level operators are present, but the proof is more elaborated; see ref. [48].) (iii) For the very same reason, UV corrections to light field propagators can be neglected [31]. (iv) Likewise, for all practical purposes in the process of matching, any heavy renormalised mass $M$ (evaluated at a scale $\mu$ equal to the physical mass) can be identified with the physical mass itself. Finally, for $m_{N} \neq 0$, this model features also the decay $N \rightarrow \nu \gamma$. Any other model fulfilling the aforementioned properties necessarily involves a larger number of degrees of freedom.

At energies $E<M \equiv \min \left\{M_{X_{E}}, M_{X_{N}}, M_{\varphi}\right\}$, this model can be described by a local EFT built upon the SM fields and $N$, also known as $\nu$ SMEFT. To leading order in the expansion in $E / M$, it is given by $\mathcal{L}_{\mathrm{SM}+N}$ (with IR parameters) and a set of dimension-six operators:

$$
\mathcal{L}_{\mathrm{EFT}}=\mathcal{L}_{\mathrm{SM}+N}^{\mathrm{IR}}+\frac{1}{\Lambda^{2}} \sum_{i} \alpha_{i} \mathcal{O}_{i}
$$

with $\alpha_{i}$ being dimensionless couplings. A basis of the operators $\mathcal{O}_{i},{ }^{4}$ obtained with the

\footnotetext{
${ }^{3}$ Indeed, any one-loop amplitude in the UV and in the EFT would read $\mathcal{M}_{\mathrm{UV}} \sim g_{\mathrm{UV}} /(4 \pi)^{2}$ and $\mathcal{M}_{\mathrm{EFT}} \sim$ $\alpha_{\mathrm{EFT}}\left[1+g_{\mathrm{EFT}} /(4 \pi)^{2}\right]$, respectively. Matching $\mathcal{M}_{\mathrm{UV}}=\mathcal{M}_{\mathrm{EFT}}$ implies therefore

$$
\alpha_{\mathrm{EFT}} \sim \frac{g_{\mathrm{UV}}}{(4 \pi)^{2}}\left[1-\frac{g_{\mathrm{EFT}}}{(4 \pi)^{2}}\right]=\frac{g_{\mathrm{UV}}}{(4 \pi)^{2}}+\mathcal{O}\left\{\frac{1}{(4 \pi)^{4}}\right\} .
$$

The last term in the right-hand side of the equation is formally of the same order as two-loop corrections and hence negligible.

${ }^{4} \mathrm{We}$ are not showing explicitly the CP counterparts of these operators, because we assume CP conservation. However, they include: $i B_{\mu \nu}\left(\bar{N} \gamma^{\mu} \partial^{\nu} N\right)$ (the one without $i$, for both the normal field strength and for the dual, is redundant), $i \mathcal{O}_{N B}, i \mathcal{O}_{N W}$ (dipole operators with the dual are redundant), $i \mathcal{O}_{L N}^{1,2,3,4}, i \mathcal{O}_{L N H}$, $i \mathcal{O}_{H N}\left(i \mathcal{O}_{N N}^{2}\right.$ is redundant) and $i \mathcal{O}_{H N e}$. Note that $\partial^{\nu} \tilde{B}_{\mu \nu}\left(\bar{N} \gamma^{\mu} N\right)$ vanishes due to the Bianchi identity. On the four-fermion side, we would have $i \mathcal{O}_{d u N e}, i \mathcal{O}_{L N L e}, i \mathcal{O}_{L N Q d}, i \mathcal{O}_{L d Q N}$ and $i \mathcal{O}_{Q u N L}$.
} 


\begin{tabular}{|c|c|c|}
\hline 0 -Higgs & 1 -Higgs & 2 -Higgs \\
\hline $\mathcal{O}_{D N}^{1}=\bar{N} \partial^{2} \not \partial N$ & $\mathcal{O}_{N B}=\bar{L} \sigma^{\mu \nu} N \tilde{H} B_{\mu \nu}, \mathcal{O}_{N W}=\bar{L} \sigma^{\mu \nu} N \sigma_{I} \tilde{H} W_{\mu \nu}^{I}$ & $\mathcal{O}_{H N}=\bar{N} \gamma^{\mu} N\left(H^{\dagger} i D_{\mu} H\right)$ \\
$\mathcal{O}_{D N}^{2}=i \tilde{B}_{\mu \nu}\left(\bar{N} \gamma^{\mu} \partial^{\nu} N\right)$ & $\mathcal{O}_{L N}^{1}=\bar{L} N D^{2} \tilde{H}, \mathcal{O}_{L N}^{2}=\bar{L} \partial_{\mu} N D^{\mu} \tilde{H}$ & $\mathcal{O}_{N N}^{2}=\bar{N} i \not N\left(H^{\dagger} H\right)$ \\
$\mathcal{O}_{D N}^{3}=\partial^{\nu} B_{\mu \nu}\left(\bar{N} \gamma^{\mu} N\right)$ & $\mathcal{O}_{L N}^{3}=i \bar{L} \sigma^{\mu \nu} \partial_{\mu} N D_{\nu} \tilde{H}, \mathcal{O}_{L N}^{4}=\bar{L}\left(\partial^{2} N\right) \tilde{H}$ & $\mathcal{O}_{H N e}=\bar{N} \gamma^{\mu} e\left(\tilde{H}^{\dagger} i D_{\mu} H\right)$ \\
\hline \multicolumn{2}{|c|}{$3-$ Higgs: $\mathcal{O}_{L N H}=\bar{L} \tilde{H} N\left(H^{\dagger} H\right)$} \\
\hline
\end{tabular}

Table 1. Relevant bosonic operators. The h.c. is implied when needed. For example, $\mathcal{O}_{D N}^{1}=$ $\bar{N} \partial^{2} \not \partial N+$ h.c.; therefore all Wilson coefficients are real.

\begin{tabular}{|ccc|}
\hline \multicolumn{3}{|c|}{$\mathcal{O}_{N N}=\left(\bar{N} \gamma_{\mu} N\right)\left(\bar{N} \gamma^{\mu} N\right)$} \\
\hline $\mathcal{O}_{e N}=\left(\bar{e} \gamma_{\mu} e\right)\left(\bar{N} \gamma^{\mu} N\right)$ & $\mathcal{O}_{u N}=\left(\bar{u} \gamma_{\mu} u\right)\left(\bar{N} \gamma^{\mu} N\right)$ \\
& $\mathcal{O}_{d N}=\left(\bar{d} \gamma_{\mu} d\right)\left(\bar{N} \gamma^{\mu} N\right)$ & $\mathcal{O}_{d u N e}=\left(\bar{d} \gamma_{\mu} u\right)\left(\bar{N} \gamma^{\mu} e\right)$ \\
\hline LLRR & $\mathcal{O}_{L N}=\left(\bar{L} \gamma_{\mu} L\right)\left(\bar{N} \gamma^{\mu} N\right)$ & $\mathcal{O}_{Q N}=\left(\bar{Q} \gamma_{\mu} Q\right)\left(\bar{N} \gamma^{\mu} N\right)$ \\
\hline \multirow{2}{*}{$\mathcal{O}_{L N L e}=(\bar{L} N) \epsilon(\bar{L} e)$} & $\mathcal{O}_{L N Q d}=(\bar{L} N) \epsilon(\bar{Q} d)$ \\
\hline LRRL & $\mathcal{O}_{L d Q N}=(\bar{L} d) \epsilon(\bar{Q} N)$ \\
\hline \multicolumn{2}{|c|}{$\mathcal{O}_{Q u N L}=(\bar{Q} u)(\bar{N} L)$} \\
\hline
\end{tabular}

Table 2. Relevant four-fermion operators.

help of BasisGen [49] (see ref. [50] for a similar code), is given in tables 1 and 2. When evaluated on shell, the operators in grey can be removed from the action by suitable field redefinitions which, up to dimension-eight effects, can be implemented by using the equations of motion [51-53]. (Redundancies due to algebraic or Fierz identities or integration by parts have been removed.) Neglecting the small $m_{N}$ and the Yukawa couplings, the relevant equations of motion of $\mathcal{L}_{\mathrm{SM}+N}$ read:

$$
\begin{aligned}
i \not \partial N & =0 \\
i \not D L & =0 \\
\left(D^{2} \tilde{H}\right)^{i} & =\mu_{H}^{2} \tilde{H}^{i}-\lambda_{H}\left(H^{\dagger} H\right) \tilde{H}^{i}, \\
\partial^{\nu} B_{\nu \mu} & =-\frac{g^{\prime}}{2}\left(i H^{\dagger} D_{\mu} H+\text { h.c. }\right)-g^{\prime} Y^{f} \bar{f} \gamma_{\mu} f,
\end{aligned}
$$

where $f$ runs over all SM $+N$ fermions. (The top Yukawa coupling is not negligible; however, its only impact would be the generation of four-fermion operators involving top quarks and $N$, for which there are no sensible searches.) As a consequence, the following 
relations hold on shell for the operators in grey:

$$
\begin{aligned}
\mathcal{O}_{D N}^{1} & =0, \\
\mathcal{O}_{D N}^{2} & =-\mathcal{O}_{D N}^{3}, \\
\mathcal{O}_{D N}^{3} & =\frac{g^{\prime}}{2} \mathcal{O}_{H N}+g^{\prime} Y^{f} \mathcal{O}_{f N}, \\
\mathcal{O}_{L N}^{1} & =\left(\mu_{H}^{2} \bar{L} \tilde{H} N+\text { h.c. }\right)-\lambda_{H} \mathcal{O}_{L N H}, \\
\mathcal{O}_{L N}^{2} & =-\mathcal{O}_{L N}^{3}, \\
\mathcal{O}_{L N}^{3} & =\left(\frac{\mu_{H}^{2}}{2} \bar{L} \tilde{H} N+\text { h.c. }\right)-\frac{\lambda_{H}}{2} \mathcal{O}_{L N H}+\frac{g^{\prime}}{8} \mathcal{O}_{N B}-\frac{g}{8} \mathcal{O}_{N W}, \\
\mathcal{O}_{L N}^{4} & =0, \\
\mathcal{O}_{N N}^{2} & =0 .
\end{aligned}
$$

As a final remark, let us note that, in light of these equations, effective operators involving $N$ do not generate any purely SMEFT operators upon using the equations of motion.

\section{Matching}

Hereafter, we assume for simplicity $M_{X_{E}}=M_{X_{N}}=M_{\varphi}=M$. Also, we focus on the regime $g_{X} \sim g_{L} \sim \lambda_{\varphi H} \ll g_{N}$, and $g_{N}>1$ (but $\lesssim 4 \pi$ to stay in the perturbative regime). This way, the mass and loop suppression in operators involving $N$ is compensated by the large $g_{N}$. On the other hand, purely SMEFT operators can be neglected.

Our process of matching consists of equating one-light-particle-irreducible amplitudes computed in both the UV and the EFT at a scale $\mu=M$ in $\overline{M S}$ with space-time dimension $d=4-2 \epsilon$. Following the discussion above, we only compute those amplitudes involving $N$. Let us also note that, by virtue of eq. (2.11), the amplitude involving just two $N$ fields, to which only this operator contributes, does not need to be computed. Likewise, due to the absence of heavy particle couplings to $e$ in the UV Lagrangian, it can be trivially seen that $\alpha_{H N e}=0$.

The operators $\mathcal{O}_{D N}^{2}$ and $\mathcal{O}_{D N}^{3}$ can be matched by computing the amplitude given by the diagrams ${ }^{5}(a)$ and $(b)$ in figure 1 . We use the momentum of the incoming $N$ and the momentum of the $B, p_{N}$ and $p_{B}$, respectively. In $\overline{M S}$ we drop terms proportional to $(1 / \epsilon+\log 4 \pi-\gamma)$, where $\gamma$ is the Euler-Mascheroni constant. The amplitudes in the UV and in the EFT to order $\mathcal{O}\left(p^{2}\right)$ read:

$$
\begin{aligned}
i \mathcal{M}_{\mathrm{UV}}= & \frac{i g^{\prime} g_{N}^{2}}{96 \pi^{2} M^{2}} \bar{u}\left(p_{N}-p_{B}\right) P_{L}\left[\gamma^{\mu}\left(p_{B}^{2}-p_{B} p_{N}+\not p_{B} \not p_{N}\right)\right. \\
& \left.-p_{B}^{\mu} \not p_{B}-p_{B}^{\mu} \not p_{N}+p_{N}^{\mu} \not \phi_{B}\right] u\left(p_{N}\right) \epsilon_{\mu}^{*}\left(p_{B}\right), \\
i \mathcal{M}_{\mathrm{EFT}}= & \frac{i}{\Lambda^{2}} \bar{u}\left(p_{N}-p_{B}\right) P_{L}\left[\gamma^{\mu}\left(\alpha_{D N}^{3} p_{B}^{2}-2 \alpha_{D N}^{2} p_{B} p_{N}+2 \alpha_{D N}^{2} \not p_{B} \not \phi_{N}\right)\right. \\
& \left.-\alpha_{D N}^{3} p_{B}^{\mu} \not p_{B}-2 \alpha_{D N}^{2} p_{B}^{\mu} \not p_{N}+2 \alpha_{D N}^{2} p_{N}^{\mu} \not p_{B}\right] u\left(p_{N}\right) \epsilon_{\mu}^{*}\left(p_{B}\right) .
\end{aligned}
$$

\footnotetext{
${ }^{5}$ All Feynman diagrams in this article are produced with the TikZ-Feynman package [54].
} 
(a)

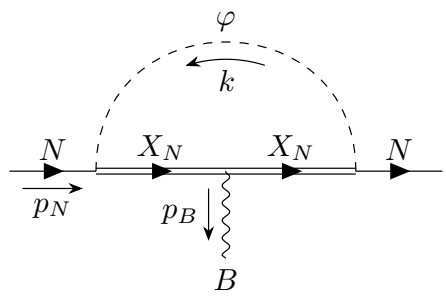

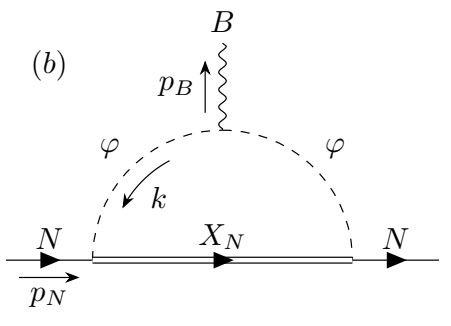

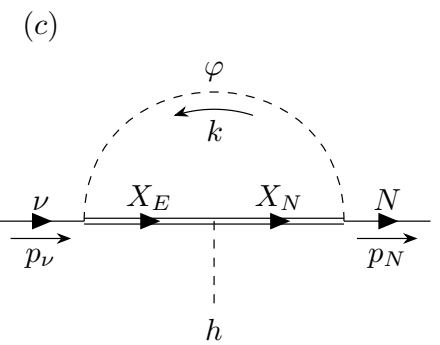

Figure 1. (a) and (b) Diagrams for the amplitude $\langle N N B\rangle$ in the UV, to which $\mathcal{O}_{D N}^{2}$ and $\mathcal{O}_{D N}^{3}$ contribute in the IR. (c) Diagram for the amplitude $\langle\nu N h\rangle$ in the UV, to which $\mathcal{O}_{L N}^{1}, \mathcal{O}_{L N}^{2}, \mathcal{O}_{L N}^{3}$ and $\mathcal{O}_{L N}^{4}$ contribute in the IR.

(a)

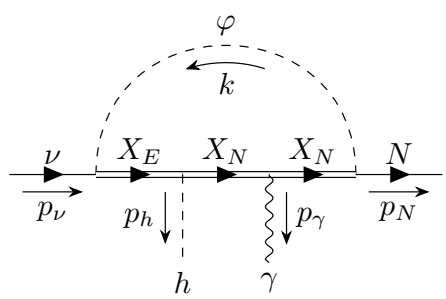

(b)

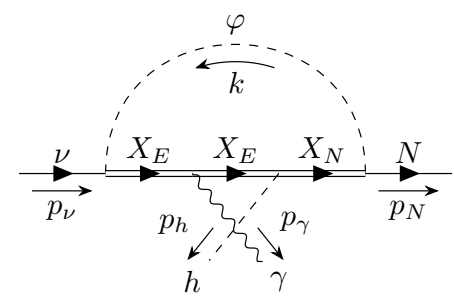

$(c)$

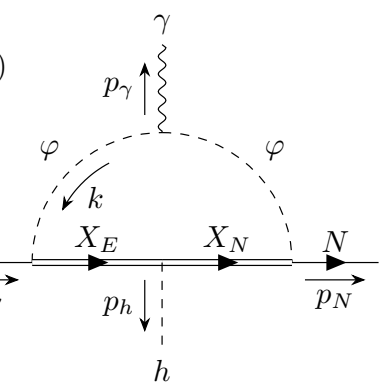

Figure 2. Diagrams for the amplitude $\langle\nu N h \gamma\rangle$ in the UV, to which $\mathcal{O}_{N B}$ and $\mathcal{O}_{N W}$ contribute in the IR.

We provide all details about the computation of this and the forthcoming UV amplitudes in appendix B.

The operators $\mathcal{O}_{L N}^{1}, \mathcal{O}_{L N}^{2}, \mathcal{O}_{L N}^{3}$ and $\mathcal{O}_{L N}^{4}$, as well as $Y_{N}$ in the IR, can be matched by computing the amplitude represented by the diagram $(c)$ in figure 1 . We take $p_{\nu}$ and $p_{N}$ as independent momenta. To order $\mathcal{O}\left(p^{2}\right)$ we have:

$$
\begin{aligned}
i \mathcal{M}_{\mathrm{UV}}= & \frac{i g_{N} g_{X} g_{L}}{96 \sqrt{2} \pi^{2} M^{2}} \bar{u}\left(p_{N}\right) P_{L}\left[6 M^{2}\left(1-\log \frac{\mu^{2}}{M^{2}}\right)-p_{\nu}^{2}-p_{N}^{2}+p_{\nu} p_{N}+\not p_{N} \not p_{\nu}\right] u\left(p_{\nu}\right), \\
i \mathcal{M}_{\mathrm{EFT}}= & \frac{i}{\sqrt{2} \Lambda^{2}} \bar{u}\left(p_{N}\right) P_{L}\left[-Y_{N} \Lambda^{2}-\alpha_{L N}^{1} p_{\nu}^{2}+\left(\alpha_{L N}^{2}-\alpha_{L N}^{1}-\alpha_{L N}^{4}\right) p_{N}^{2}\right. \\
& \left.+\left(2 \alpha_{L N}^{1}-\alpha_{L N}^{2}+\alpha_{L N}^{3}\right) p_{\nu} p_{N}-\alpha_{L N}^{3} \not p_{N} \not p_{\nu}\right] u\left(p_{\nu}\right) .
\end{aligned}
$$

The other two operators involving a single Higgs field are $\mathcal{O}_{N B}$ and $\mathcal{O}_{N W}$. They can be matched by computing the amplitude represented by the diagrams in figure 2. Taking $p_{\gamma}$, $p_{h}$ and $p_{N}$ as independent momenta, the results in the UV and in the EFT up to order 

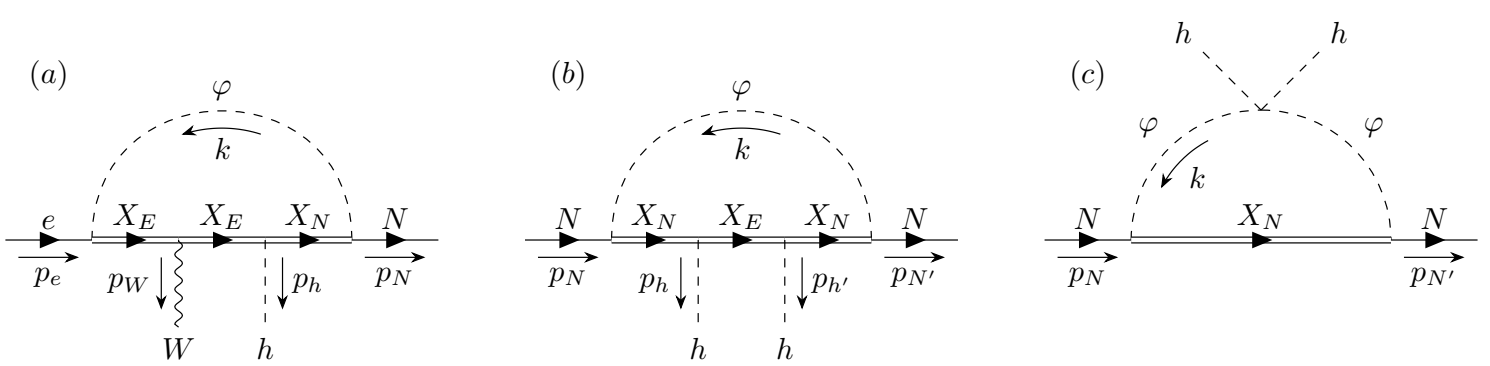

Figure 3. (a) Diagram for the amplitude $\langle e N W h\rangle$ in the UV, to which $\mathcal{O}_{L N}^{1}, \mathcal{O}_{L N}^{2}, \mathcal{O}_{L N}^{3}$ and $\mathcal{O}_{N W}$ contribute in the IR. $(b)$ and $(c)$ Diagrams for the amplitude $\langle N N h h\rangle$ in the UV, to which $\mathcal{O}_{N N}^{2}$ contributes in the IR. (Note that, despite not explicitly shown, diagram (b) but with the two Higgses exchanged is also present.)

$\mathcal{O}(p)$ read:

$$
\begin{aligned}
i \mathcal{M}_{\mathrm{UV}} & =\frac{i g_{L} g_{X} g_{N} e}{96 \sqrt{2} \pi^{2} M^{2}} \bar{u}\left(p_{N}\right) P_{L}\left[\gamma^{\mu} \not p_{\gamma}-p_{\gamma}^{\mu}\right] u\left(p_{\nu}\right) \epsilon_{\mu}^{*}\left(p_{\gamma}\right) \\
i \mathcal{M}_{\mathrm{EFT}} & =\frac{\sqrt{2} i}{\Lambda^{2}}\left(c_{W} \alpha_{N B}+s_{W} \alpha_{N W}\right) \bar{u}\left(p_{N}\right) P_{L}\left[\gamma^{\mu} \not p_{\gamma}-p_{\gamma}^{\mu}\right] u\left(p_{\nu}\right) \epsilon_{\mu}^{*}\left(p_{\gamma}\right)
\end{aligned}
$$

Here $c_{W} \equiv \cos \theta_{W}$ and $s_{W} \equiv \sin \theta_{W}$, with $\theta_{W}$ being the weak mixing angle. (Let us emphasise that in our convention, $W_{\mu}^{3}=c_{W} Z_{\mu}+s_{W} A_{\mu}, B_{\mu}=c_{W} A_{\mu}-s_{W} Z_{\mu}$.) This amplitude was also computed previously in ref. [15] (see appendix therein). Still, one more amplitude needs to be computed in order to completely fix the Wilson coefficients of the one-Higgs operators. We choose that represented by the diagram $(a)$ in figure 3. Taking $p_{h}, p_{W}$ and $p_{N}$ as independent momenta, we have to order $\mathcal{O}(p)$ :

$$
\begin{aligned}
i \mathcal{M}_{\mathrm{UV}}= & \frac{i g_{N} g_{X} g_{L} g}{192 \pi^{2} M^{2}} \bar{u}\left(p_{N}\right) P_{L}\left[p_{N}^{\mu}-2 p_{h}^{\mu}-p_{W}^{\mu}-\gamma^{\mu} \not p_{N}\right] u\left(p_{e}\right) \epsilon_{\mu}^{*}\left(p_{W}\right), \\
i \mathcal{M}_{\mathrm{EFT}}= & \frac{i g}{2 \Lambda^{2}} \bar{u}\left(p_{N}\right) P_{L}\left[-\left(\alpha_{L N}^{2}+\alpha_{L N}^{3}\right) p_{N}^{\mu}-2 \alpha_{L N}^{1} p_{h}^{\mu}-\left(4 \frac{\alpha_{N W}}{g}+\alpha_{L N}^{1}\right) p_{W}^{\mu}\right. \\
& \left.+\alpha_{L N}^{3} \gamma^{\mu} \not p_{N}+4 \frac{\alpha_{N W}}{g} \gamma^{\mu} \not p_{W}\right] u\left(p_{e}\right) \epsilon_{\mu}^{*}\left(p_{W}\right) .
\end{aligned}
$$

The operator $\mathcal{O}_{N N}^{2}$ can be matched by computing the amplitude represented by the diagrams $(b)$ and $(c)$ in figure 3 , while the operator $\mathcal{O}_{H N}$ by computing the diagram $(a)$ in figure 4. (It might seem that $\mathcal{O}_{H N}$ also contributes to the former amplitude; however, only its $\mathrm{CP}$ counterpart $i \mathcal{O}_{H N}$, which we do not need to consider, does it.) The first one reads, to order $\mathcal{O}(p)$ :

$$
\begin{aligned}
i \mathcal{M}_{\mathrm{UV}} & =-\frac{i g_{N}^{2} \lambda_{\varphi H}}{96 \pi^{2} M^{2}} \bar{u}\left(p_{N^{\prime}}\right) P_{L}\left[\not p_{N}+\not p_{N^{\prime}}\right] u\left(p_{N}\right), \\
i \mathcal{M}_{\mathrm{EFT}} & =\frac{i}{\Lambda^{2}} \alpha_{N N}^{2} \bar{u}\left(p_{N^{\prime}}\right) P_{L}\left[\not p_{N}+\not p_{N^{\prime}}\right] u\left(p_{N}\right) .
\end{aligned}
$$



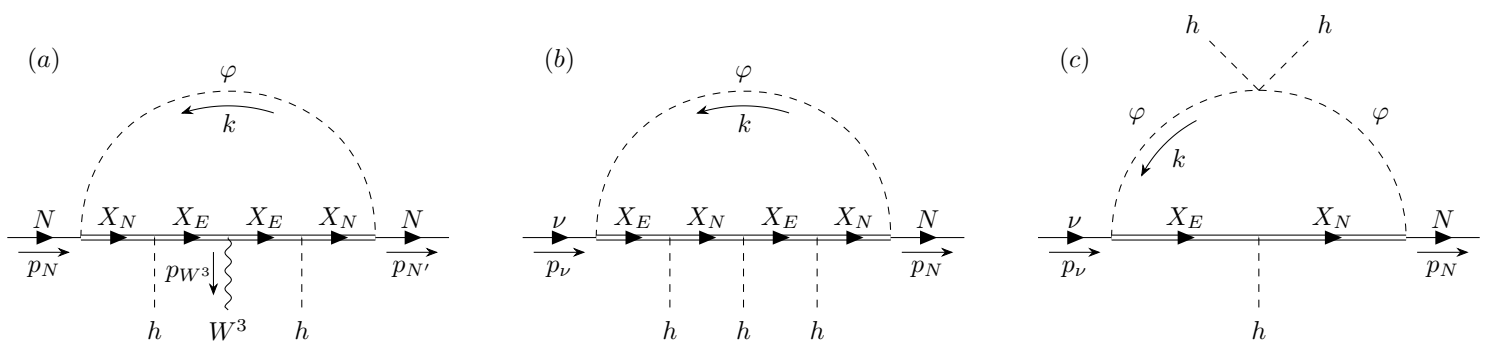

Figure 4. (a) Diagram for the amplitude $\left\langle N N W^{3} h h\right\rangle$ in the UV, to which the operator $\mathcal{O}_{H N}$ contributes in the IR. (b) and (c) Diagrams for the amplitude $\langle\nu N h h h\rangle$ in the UV, to which $\mathcal{O}_{L N H}$ contributes in the IR. (Note that, despite not explicitly shown, all these diagrams but with the corresponding Higgs legs exchanged are also present.)

In the UV and EFT to zero momentum, the second aforementioned amplitude reads:

$$
\begin{aligned}
i \mathcal{M}_{\mathrm{UV}} & =\frac{i g g_{N}^{2} g_{X}^{2}}{96 \pi^{2} M^{2}} \bar{u}\left(p_{N^{\prime}}\right) P_{L} \gamma^{\mu} u\left(p_{N}\right) \epsilon_{\mu}^{*}\left(p_{W^{3}}\right), \\
i \mathcal{M}_{\mathrm{EFT}} & =-\frac{i \alpha_{H N} g}{\Lambda^{2}} \bar{u}\left(p_{N^{\prime}}\right) P_{L} \gamma^{\mu} u\left(p_{N}\right) \epsilon_{\mu}^{*}\left(p_{W^{3}}\right) .
\end{aligned}
$$

The operator $\mathcal{O}_{L N H}$ can be matched by computing the amplitude depicted by the diagrams $(b)$ and $(c)$ in figure 4 . To zero momentum, the amplitudes in the UV and in the EFT are given by [15]:

$$
\begin{aligned}
i \mathcal{M}_{\mathrm{UV}} & =\frac{i g_{N} g_{X} g_{L}}{32 \sqrt{2} \pi^{2} M^{2}}\left(\lambda_{\varphi H}-g_{X}^{2}\right) \bar{u}\left(p_{N}\right) P_{L} u\left(p_{\nu}\right), \\
i \mathcal{M}_{\mathrm{EFT}} & =\frac{3 i \alpha_{L N H}}{\sqrt{2} \Lambda^{2}} \bar{u}\left(p_{N}\right) P_{L} u\left(p_{\nu}\right) .
\end{aligned}
$$

On the side of four-fermion operators, the only such non-vanishing interactions (before using the equations of motion) are $\mathcal{O}_{N N}$ and $\mathcal{O}_{L N}$. They can be matched by computing the amplitudes depicted by the diagrams $(a)$ and $(b)$ in figure 5 , respectively. The UV and EFT expressions for each amplitude to zero momentum read, respectively:

$$
\begin{aligned}
i \mathcal{M}_{\mathrm{UV}} & =-\frac{i g_{N}^{4}}{96 \pi^{2} M^{2}}\left[\bar{u}\left(p_{3}\right) \gamma^{\mu} P_{R} u\left(p_{1}\right)\right]\left[\bar{u}\left(p_{4}\right) \gamma_{\mu} P_{R} u\left(p_{2}\right)\right], \\
i \mathcal{M}_{\mathrm{EFT}} & =\frac{4 i \alpha_{N N}}{\Lambda^{2}}\left[\bar{u}\left(p_{3}\right) \gamma^{\mu} P_{R} u\left(p_{1}\right)\right]\left[\bar{u}\left(p_{4}\right) \gamma_{\mu} P_{R} u\left(p_{2}\right)\right],
\end{aligned}
$$

and

$$
\begin{aligned}
i \mathcal{M}_{\mathrm{UV}} & =-\frac{i g_{N}^{2} g_{L}^{2}}{192 \pi^{2} M^{2}}\left[\bar{u}\left(p_{\nu^{\prime}}\right) \gamma^{\mu} P_{L} u\left(p_{\nu}\right)\right]\left[\bar{u}\left(p_{N^{\prime}}\right) \gamma_{\mu} P_{R} u\left(p_{N}\right)\right], \\
i \mathcal{M}_{\mathrm{EFT}} & =\frac{i \alpha_{L N}}{\Lambda^{2}}\left[\bar{u}\left(p_{\nu^{\prime}}\right) \gamma^{\mu} P_{L} u\left(p_{\nu}\right)\right]\left[\bar{u}\left(p_{N^{\prime}}\right) \gamma_{\mu} P_{R} u\left(p_{N}\right)\right] .
\end{aligned}
$$

By equating all UV amplitudes to their IR counterparts, we end up with 23 equations (including $\alpha_{H N e}=0$ ) for 15 unknowns; the redundancies reflect the gauge symmetries. ${ }^{6}$

\footnotetext{
${ }^{6}$ Note, for example, that the contributions of $\mathcal{O}_{L N}^{1}$ to the amplitudes $\langle\nu N h\rangle$ and $\langle e N W h\rangle$ are correlated, because both the $\mathcal{O}\left(p^{2}\right)$ Higgs piece as well as the $W H$ interaction come from $D^{2} \tilde{H}$. Thus, when matching e.g. the $p_{\nu}^{2}$ part of eqs. (3.3) and (3.4) one gets $\alpha_{L N}^{1}=\left(g_{N} g_{X} g_{L}\right) /\left(96 \pi^{2}\right) \times(\Lambda / M)^{2}$; exactly the same as matching the $p_{h}^{\mu}$ piece of eqs. (3.7) and (3.8).
} 


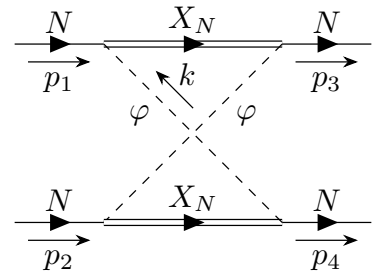

(a)

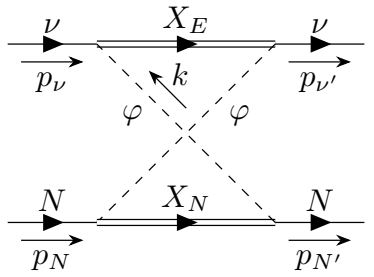

(b)

Figure 5. (a) Diagram for the amplitude $\langle N N N N\rangle$ in the UV, to which $\mathcal{O}_{N N}$ contributes in the IR. (Note that, despite not explicitly shown, this diagram but with the two outgoing $N$ s exchanged also exists.) (b) Diagram for the amplitude $\langle\nu \nu N N\rangle$ in the UV, to which $\mathcal{O}_{L N}$ contributes in the IR.

Neglecting the running from the scale $\mu=M$ to $\mu=v$, the following identities hold off shell at the EW scale (other Wilson coefficients vanish):

$$
Y_{N}^{\mathrm{IR}}=Y_{N}^{\mathrm{UV}}-\frac{g_{L} g_{X} g_{N}}{16 \pi^{2}},
$$

$$
\begin{aligned}
\frac{\alpha_{D N}^{2}}{\Lambda^{2}} & =\frac{e g_{N}^{2}}{192 \pi^{2} c_{W} M^{2}}, \\
\frac{\alpha_{N B}}{\Lambda^{2}} & =\frac{e g_{L} g_{X} g_{N}}{192 \pi^{2} c_{W} M^{2}}, \\
\frac{\alpha_{L N}^{3}}{\Lambda^{2}} & =-\frac{g_{L} g_{X} g_{N}}{96 \pi^{2} M^{2}}, \\
\frac{\alpha_{N N}^{2}}{\Lambda^{2}} & =-\frac{\lambda_{\varphi H} g_{N}^{2}}{96 \pi^{2} M^{2}}, \\
\frac{\alpha_{N N}}{\Lambda^{2}} & =-\frac{g_{N}^{4}}{384 \pi^{2} M^{2}},
\end{aligned}
$$

$$
\begin{aligned}
\frac{\alpha_{D N}^{3}}{\Lambda^{2}} & =\frac{e g_{N}^{2}}{96 \pi^{2} c_{W} M^{2}}, \\
\frac{\alpha_{L N}^{1}}{\Lambda^{2}} & =\frac{g_{L} g_{X} g_{N}}{96 \pi^{2} M^{2}}, \\
\frac{\alpha_{H N}}{\Lambda^{2}} & =-\frac{g_{X}^{2} g_{N}^{2}}{96 \pi^{2} M^{2}}, \\
\frac{\alpha_{L N H}}{\Lambda^{2}} & =\frac{g_{L} g_{X} g_{N}}{96 \pi^{2} M^{2}}\left(\lambda_{\varphi H}-g_{X}^{2}\right), \\
\frac{\alpha_{L N}}{\Lambda^{2}} & =-\frac{g_{L}^{2} g_{N}^{2}}{192 \pi^{2} M^{2}},
\end{aligned}
$$

where $e=\sqrt{4 \pi \alpha}$, and $\alpha \approx 1 / 137$ stands for the electromagnetic fine-structure constant. Finally, upon using the equations of motion, eqs. (2.11)-(2.18), the following relations hold on shell (other Wilson coefficients vanish):

$$
\begin{aligned}
& Y_{N}^{\mathrm{IR}}=Y_{N}^{\mathrm{UV}}-\frac{g_{L} g_{X} g_{N}}{16 \pi^{2}}\left(1+\frac{m_{h}^{2}}{24 M^{2}}\right), \\
& \frac{\alpha_{N B}}{\Lambda^{2}}=\frac{e g_{L} g_{X} g_{N}}{256 \pi^{2} c_{W} M^{2}}, \\
& \frac{\alpha_{H N}}{\Lambda^{2}}=\frac{g_{N}^{2}\left(e^{2}-4 c_{W}^{2} g_{X}^{2}\right)}{384 \pi^{2} c_{W}^{2} M^{2}}, \\
& \frac{\alpha_{L N}}{\Lambda^{2}}=-\frac{g_{N}^{2}\left(e^{2}+2 c_{W}^{2} g_{L}^{2}\right)}{384 \pi^{2} c_{W}^{2} M^{2}}, \\
& \frac{\alpha_{N N}}{\Lambda^{2}}=-\frac{g_{N}^{4}}{384 \pi^{2} M^{2}}, \\
& \frac{\alpha_{u N}}{\Lambda^{2}}=\frac{e^{2} g_{N}^{2}}{288 \pi^{2} c_{W}^{2} M^{2}}, \\
& \frac{\alpha_{N W}}{\Lambda^{2}}=\frac{e g_{L} g_{X} g_{N}}{768 \pi^{2} s_{W} M^{2}}, \\
& \frac{\alpha_{L N H}}{\Lambda^{2}}=-\frac{g_{L} g_{X} g_{N}}{192 \pi^{2} M^{2}}\left[\frac{m_{h}^{2}}{v^{2}}+2\left(g_{X}^{2}-\lambda_{\varphi H}\right)\right] \text {, } \\
& \frac{\alpha_{e N}}{\Lambda^{2}}=-\frac{e^{2} g_{N}^{2}}{192 \pi^{2} c_{W}^{2} M^{2}}, \\
& \frac{\alpha_{Q N}}{\Lambda^{2}}=\frac{e^{2} g_{N}^{2}}{1152 \pi^{2} c_{W}^{2} M^{2}}, \\
& \frac{\alpha_{d N}}{\Lambda^{2}}=-\frac{e^{2} g_{N}^{2}}{576 \pi^{2} c_{W}^{2} M^{2}} \text {. }
\end{aligned}
$$


For convenience, let us also define $\mathcal{O}_{N A}=c_{W} \mathcal{O}_{N B}+s_{W} \mathcal{O}_{N W}$ and $\mathcal{O}_{N Z}=c_{W} \mathcal{O}_{N W}-$ $s_{W} \mathcal{O}_{N B}$. For the coefficients of these operators we obtain:

$$
\frac{\alpha_{N A}}{\Lambda^{2}}=\frac{e g_{L} g_{X} g_{N}}{192 \pi^{2} M^{2}}, \quad \frac{\alpha_{N Z}}{\Lambda^{2}}=\frac{e g_{L} g_{X} g_{N}\left(1-4 s_{W}^{2}\right)}{768 \pi^{2} s_{W} c_{W} M^{2}} .
$$

Finally, let us emphasise that the coupling constants of renormalisable operators in the EFT can also be written in terms of the UV couplings; $g_{\mathrm{IR}}=g_{\mathrm{UV}}+g_{\mathrm{UV}}^{\prime} /\left(16 \pi^{2}\right)$. However, one can always reabsorb the one-loop corrections by redefining $g_{\mathrm{UV}} \rightarrow g_{\mathrm{UV}}-g_{\mathrm{UV}}^{\prime} /\left(16 \pi^{2}\right)$. This redefinition would propagate to all $\alpha$ couplings, but the impact would be formally of two-loop order. Still, for the sake of completeness, we have computed all renormalisable EFT terms in appendix C.

\section{Sterile neutrino phenomenology}

In the process of matching we have neglected $m_{N}$. The only effect of $m_{N} \neq 0$ would appear in the dimension-five operator $\overline{N^{c}} N H^{\dagger} H$ suppressed not only by the loop factor but also by $m_{N} / M$, namely by $\sim 10^{-3}$ if $m_{N} \sim 1 \mathrm{GeV}$ and $M \sim 1 \mathrm{TeV}$. (The operator $\overline{N^{c}} \sigma^{\mu \nu} N B_{\mu \nu}$ vanishes in this case because $N$ is Majorana.) However, $m_{N} \neq 0$ has a huge impact on the phenomenology of $N$, because it allows the latter to decay into $\nu \gamma$. The corresponding decay width must be computed after EWSB, namely in the $\nu$ LEFT, obtained first by matching the $\nu \mathrm{SMEFT}$ at the EW scale and after running down to $\sim m_{N}$. The full list of $\nu$ LEFT operators involving $N$ is given in table 3 in appendix $\mathrm{D}$. The operator that triggers the decay of $N$ is $\mathcal{O}_{N \gamma}$. For completeness, though, we provide tree-level matching of all $\nu$ SMEFT operators to all $\nu$ LEFT ones in eqs. (D.1)-(D.24). The one-loop running of all $\nu$ LEFT operators generated in our setup, including $\mathcal{O}_{N \gamma}$, is also given in the same appendix. Altogether, we have:

$$
\Gamma(N \rightarrow \nu \gamma) \approx \frac{m_{N}^{3} \alpha_{N \gamma}^{2}(v)}{2 \pi v^{2}}\left(1-\frac{5 e^{2}}{9 \pi^{2}} \log \frac{v}{m_{N}}\right)^{2} .
$$

For simplicity, let us fix $Y_{N}^{\mathrm{UV}}$ such that $Y_{N}^{\mathrm{IR}}=\alpha_{L N H} v^{2} /\left(2 \Lambda^{2}\right)$; see eq. (3.30). This way, the mixing between $N$ and $\nu$ vanishes strictly. (Note that the sole important effect of this mixing would be inducing a Majorana neutrino dipole moment for $\nu$; this vanishes however in our case due to lepton flavour conservation [15].)

Different experiments constrain the parameter space under study. The relevant observables can be all computed directly in the $\nu$ SMEFT. (In quoting the following bounds we have set $\Lambda=1 \mathrm{TeV}$.) We have first $\mathcal{B}(Z \rightarrow \nu \nu \gamma \gamma)$. Experimentally it is bounded to be $<3.1 \times 10^{-6}[55]$. In our context this branching ratio is given by (note that for $m_{N} \sim 1 \mathrm{GeV}$, $\mathcal{B}(N \rightarrow \nu \gamma) \approx 1[56]):$

$$
\begin{aligned}
\mathcal{B}(Z \rightarrow \nu \nu \gamma \gamma) \approx \mathcal{B}(Z \rightarrow N N) & \approx \frac{1}{\Gamma_{Z}^{\mathrm{SM}}} \frac{m_{Z}^{3} v^{2}}{24 \pi \Lambda^{4}} \alpha_{H N}^{2} \\
& \approx 2.7 \times 10^{-10} g_{N}^{4}\left(0.029-g_{X}^{2}\right)^{2} \frac{\mathrm{TeV}^{4}}{M^{4}}
\end{aligned}
$$


with $\Gamma_{Z}^{\mathrm{SM}} \approx 2.5 \mathrm{GeV}$. This bound implies in turn a limit on $\alpha_{H N}<0.11$, which is ultimately the most stringent constrain on $\left(M, g_{N}\right)$. Other subleading constraints include: (i) $\mathcal{B}(Z \rightarrow$ $\nu \nu \gamma$ ), experimentally bounded to be $<3.2 \times 10^{-6}$ [57], which implies $\alpha_{N Z}<0.081$; (ii) the measurement of the total $W$ boson width, $\Gamma_{W}^{\text {total }}=2.085 \pm 0.042 \mathrm{GeV}$, which however does not constrain $\alpha_{H N e}$ more than a theoretical perturbativity bound implying $\alpha_{H N e}<4 \pi$; (iii) the bound on $\alpha_{N A}<0.88$ [15] as obtained from LHC searches for events with one photon and missing energy [58]. (Bounds on $\alpha_{N A}$ obtained from the study of differential Drell-Yan distributions at the LHC, mediated by both neutral and charged currents, using Contur [59] are weaker [15].) The bound on $\alpha_{N A}$ can be improved to $\alpha_{N A}<0.36$ by searches for $h \rightarrow \gamma \gamma+E_{T}^{\text {miss }}$, as proposed in ref. [15]. This value, however, still leads to a very weak constrain on $\left(M, g_{N}\right)$.

Four-fermion interactions could be bounded at the LHC in searches for $p p \rightarrow \ell \gamma+E_{T}^{\text {miss }}$. However, we are not aware of any such search; a preliminary phenomenological study has been provided in ref. [60]. Interestingly though, it has been shown that searches for Higgs decaying to a single photon and missing energy could test $\mathcal{B}(h \rightarrow \nu N)>1.2 \times 10^{-4}[15]$. Noticing that

$$
\begin{aligned}
\mathcal{B}(h \rightarrow \nu N) & \approx \frac{1}{\Gamma_{h}^{S M}} \frac{m_{h} v^{4}}{16 \pi \Lambda^{4}} \alpha_{L N H}^{2} \\
& \approx 2.5 \times 10^{-6}\left(g_{L} g_{N} g_{X}\right)^{2}\left(0.13+g_{X}^{2}-\lambda_{\varphi H}\right)^{2} \frac{\mathrm{TeV}^{4}}{M^{4}}
\end{aligned}
$$

with $\Gamma_{h}^{S M} \approx 4 \mathrm{MeV}$, the corresponding limit on $\alpha_{L N H}$ reads $\alpha_{L N H}<7.3 \times 10^{-3}$. We show in figure 6 that, when translated to the plane $\left(M, g_{N}\right)$, this signal overcomes often the constraint from $\alpha_{H N}$.

If lepton number is exactly conserved, i.e. in particular, $m_{N}=0, N$ is just the $\mathrm{RH}$ component of the SM neutrino, which would be Dirac. In this case, the very stringent bounds on the neutrino dipole moment [61] can be only satisfied if $g_{X} \approx 0$ (or $g_{L} \approx 0$ ). Accordingly, only the operator $\mathcal{O}_{H N}$ and the four-fermions $\mathcal{O}_{L N}, \mathcal{O}_{e N}, \mathcal{O}_{Q N}, \mathcal{O}_{u N}$ and $\mathcal{O}_{d N}$ as well as $\mathcal{O}_{N N}$ could survive, see eqs. (3.30)-(3.40). The former enhances the $Z$ decay into invisible, but the corresponding limit on $\left(M, g_{N}\right)$ is very weak. Likewise, the bounds on the four-fermion operators involving quarks and charged leptons are of order $\alpha / \Lambda^{2} \lesssim 1 \mathrm{TeV}^{-2}$ [13], and therefore they are not stringent in this setup in which all operators arise at loop level, and hence the effective scale $\Lambda$ is rather $\Lambda \sim 4 \pi M$.

Finally, we are not aware of any significant bound on $\mathcal{O}_{N N}$. As things stand, this scenario is very much unconstrained in light of current data, even for $M \sim$ few hundreds $\mathrm{GeV}$. (In this respect, let us also emphasise that direct LHC searches for singly charged scalars and vector-like leptons, which are present in our UV model, do not constrain this range of masses [62-65].)

In the lepton number conserving case, if instead of a single $N$ we have three copies (in which case all neutrinos would be Dirac), with a priori flavour-generic couplings, the offdiagonal dipole operators would induce decays of the SM neutrinos, $\nu_{j} \rightarrow \nu_{i} \gamma$. For massless 

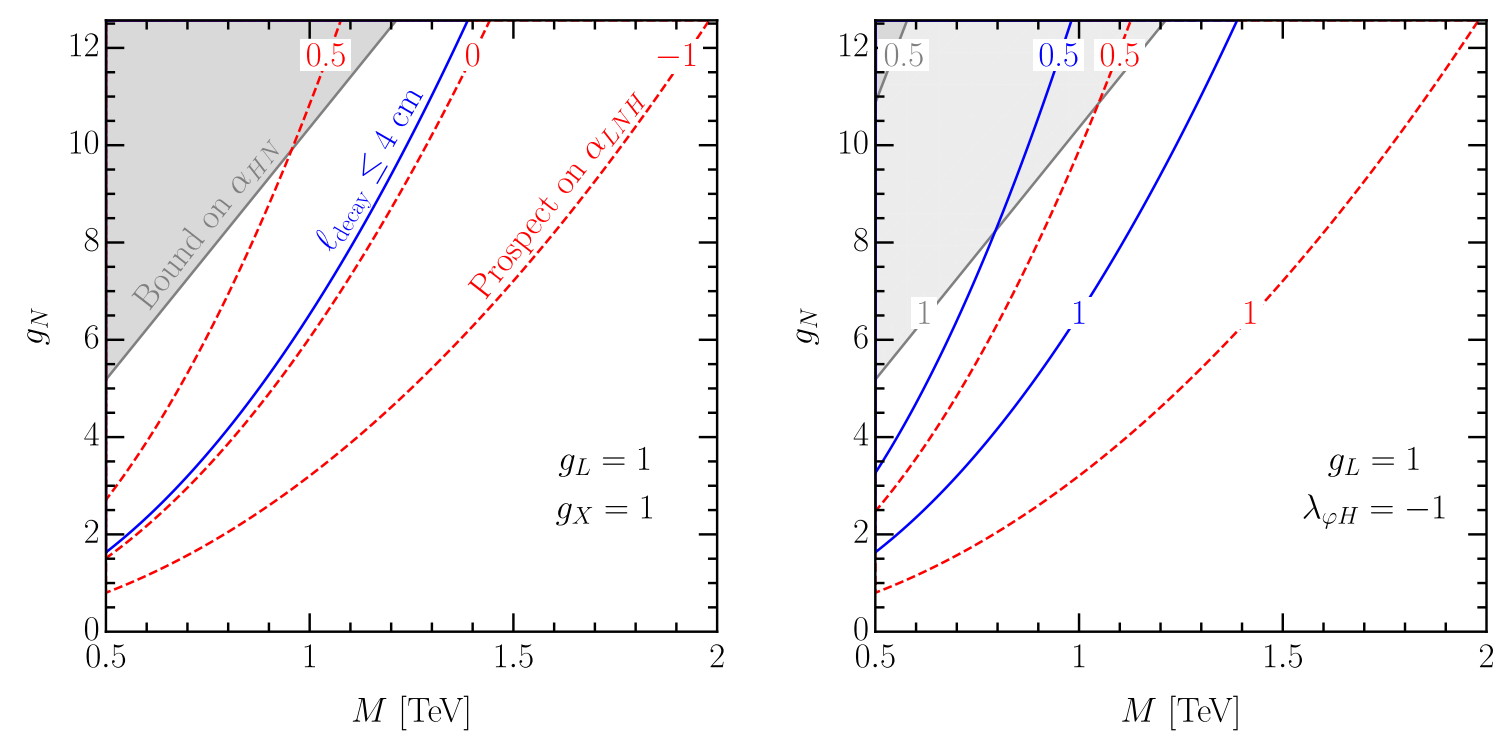

Figure 6. Constraints in the plane $\left(M, g_{N}\right)$ derived from the bounds on the EFT coefficients summarised in the text. In the region above the blue line, $N$ with $m_{N}=1 \mathrm{GeV}$ decays within $4 \mathrm{~cm}$. The actual bound on $\alpha_{H N}$ from $Z \rightarrow \nu \nu \gamma \gamma$ and the prospective bound on $\alpha_{L N H}$ from the $h \rightarrow \gamma+E_{T}^{\text {miss }}$ analysis proposed in ref. [15] have been translated to the constraints in the plane $\left(M, g_{N}\right)$ assuming $g_{X}=1$ and $\lambda_{\varphi H}=-1,0$ and 0.5 (left) as well as $\lambda_{\varphi H}=-1$ and $g_{X}=0.5$ and 1 (right).

neutrino in the final state and neglecting running, the corresponding decay width reads

$$
\Gamma\left(\nu_{j} \rightarrow \nu_{i} \gamma\right)=\frac{m_{j}^{3} v^{2}}{8 \pi \Lambda^{4}} \alpha_{N A}^{2}=\frac{m_{j}^{3} v^{2}}{8 \pi M^{4}}\left(\frac{e g_{L} g_{X} g_{N}}{192 \pi^{2}}\right)^{2},
$$

where $m_{j}$ is the mass of $\nu_{j}$. The lower limit on the neutrino lifetime is $\tau \gtrsim 10^{20} \mathrm{~s}[55,66]$. Assuming $m_{j} \approx 0.1 \mathrm{eV}$ and $\mathcal{O}(1)$ couplings, we obtain $M \gtrsim 0.3 \mathrm{TeV}$, and up to $\sqrt{4 \pi}$ times more stringent constraint if $g_{N}$ is significantly larger than 1 , as we have been assuming in this work. Still, as in the previous case, this bound can be avoided if e.g. $g_{X} \ll 1$.

\section{Conclusions}

In this paper, we have considered a very simple extension of the SM involving a light $\mathrm{RH}$ neutrino $N$ (which can be well the RH part of any of the SM neutrinos if they are Dirac, or a new Majorana neutrino), two heavy fermionic fields and one heavy scalar field, all of them colourless. In the IR, this theory can be described by the $\nu$ SMEFT. We have shown that, if $N$ is Majorana, new Higgs decays not yet studied experimentally at the LHC can test this model better than other studies already performed at low-energy facilities; most importantly, searches for $Z \rightarrow \nu \nu \gamma \gamma$.

We note that, although this observation is relatively straightforward in the generic EFT, because constrained operators can be set to zero independently of those triggering the signal of interest, this is highly non-trivial in the EFT obtained in this model, in which all Wilson coefficients depend on solely four arbitrary couplings. The fact that the signal 
of interest is not in conflict with the present constraints in such a simple UV completion of the $\nu$ SMEFT, strengthens the motivation for novel searches in the Higgs sector.

We have provided a complete calculation of one-loop matching in the diagrammatic approach, obtained upon computing the same one-light-particle-irreducible off-shell amplitudes in the UV and in the IR. This complements the very few examples of one-loop matching in the literature, and it is expected that our results will allow faster progress in the automation of tools in this respect [31, 32]. As a byproduct of this work, we have also obtained a complete (off-shell) basis in the $\nu$ SMEFT, the tree-level matching of the $\nu$ SMEFT onto the low-energy version (in which the top quark, the Higgs and the $W$ and $Z$ bosons are integrated out), that we dubbed $\nu$ LEFT; as well as some one-loop anomalous dimensions in the latter EFT. We leave the computation of the full RGE anomalous dimension matrix in the $\nu$ LEFT and in the $\nu$ SMEFT for future work.

\section{Acknowledgments}

We are grateful to Jose Santiago for helpful discussions. MC is supported by the Spanish MINECO under the Juan de la Cierva programme.

\section{A Mathematical tools}

We have used the following master integrals:

$$
\begin{aligned}
\int \frac{\mathrm{d}^{d} k}{(2 \pi)^{d}} \frac{1}{\left(k^{2}-M^{2}\right)^{n}}= & \frac{(-1)^{n} i}{(4 \pi)^{d / 2}} \frac{\Gamma(n-d / 2)}{\Gamma(n)} \frac{1}{M^{2 n-d}}=A_{n}, \\
\int \frac{\mathrm{d}^{d} k}{(2 \pi)^{d}} \frac{k^{\mu} k^{\nu}}{\left(k^{2}-M^{2}\right)^{n}}= & \frac{\underbrace{\frac{1}{2} \frac{(-1)^{n-1} i}{(4 \pi)^{d / 2}} \frac{\Gamma(n-d / 2-1)}{\Gamma(n)} \frac{1}{M^{2 n-d-2}}}_{B_{n}} g^{\mu \nu},}{C_{n}} \\
\int \frac{\mathrm{d}^{d} k}{(2 \pi)^{d}} \frac{k^{\mu} k^{\nu} k^{\rho} k^{\sigma}}{\left(k^{2}-M^{2}\right)^{n}}= & \overbrace{\frac{1}{4} \frac{(-1)^{n} i}{(4 \pi)^{d / 2}} \frac{\Gamma(n-d / 2-2)}{\Gamma(n)} \frac{1}{M^{2 n-d-4}}} \\
& \times\left(g^{\mu \nu} g^{\rho \sigma}+g^{\mu \rho} g^{\nu \sigma}+g^{\mu \sigma} g^{\nu \rho}\right) .
\end{aligned}
$$

Here $d$ is the space-time dimension. For expansion in an external momentum $p$ we have:

$$
\frac{1}{(k+p)^{2}-M^{2}}=\frac{1}{k^{2}-M^{2}}\left[1-\frac{2 k p+p^{2}}{k^{2}-M^{2}}+\frac{4(k p)^{2}}{\left(k^{2}-M^{2}\right)^{2}}\right]+\mathcal{O}\left(p^{3}\right) .
$$

Finally, we have also made use of the following algebraic identities:

$$
\begin{aligned}
\epsilon^{\mu \sigma \rho \nu} p_{1 \rho} p_{2 \nu} \gamma_{\sigma} \gamma_{5} & =i\left(\gamma^{\mu} \not p_{2} p_{1}+p_{1}^{\mu} p_{2}-p_{2}^{\mu} \not p_{1}-\gamma^{\mu} p_{1} p_{2}\right) \\
{\left[D_{\mu}, D_{\nu}\right] } & =-i g^{\prime} Y B_{\mu \nu}-i g T^{I} W_{\mu \nu}^{I} .
\end{aligned}
$$




\section{B Details of computation of the UV amplitudes}

\section{B.1 Amplitude for one $B$ and no Higgs bosons}

This amplitude in the UV is given by the diagrams $(a)$ and $(b)$ in figure 1 . We have:

$$
\begin{aligned}
i \mathcal{M}_{\mathrm{UV}}^{a}= & -g^{\prime} g_{N}^{2} \bar{u}\left(p_{N}-p_{B}\right) P_{L}\left\{\mu^{4-d} \int \frac{\mathrm{d}^{d} k}{(2 \pi)^{d}} \frac{1}{D^{3}}\left(\not p_{N}-\not p_{B}+\not k+M\right) \gamma^{\mu}\left(\not p_{N}+\not k+M\right)\right. \\
& \times\left[1-\frac{2\left(p_{N}-p_{B}\right) k+\left(p_{N}-p_{B}\right)^{2}}{D}+\frac{4\left[k\left(p_{N}-p_{B}\right)\right]^{2}}{D^{2}}\right] \\
& \left.\times\left[1-\frac{2 p_{N} k+p_{N}^{2}}{D}+\frac{4\left(k p_{N}\right)^{2}}{D^{2}}\right]\right\} P_{R} u\left(p_{N}\right) \epsilon_{\mu}^{*}\left(p_{B}\right) \\
= & -g^{\prime} g_{N}^{2} \bar{u}\left(p_{N}-p_{B}\right) P_{L}\left\{\left[\mu^{2 \epsilon}(2 \epsilon-2) B_{3}+M^{2} A_{3}\right] \gamma^{\mu}\right. \\
& +\left[12 B_{4}-A_{3}-48 C_{5}-2 M^{2} A_{4}+12 M^{2} B_{5}\right] \gamma^{\mu} p_{N}^{2} \\
& +\left[4 B_{4}-16 C_{5}-M^{2} A_{4}+4 M^{2} B_{5}\right] \gamma^{\mu} p_{B}^{2} \\
& +\left[48 C_{5}-8 B_{4}+2 M^{2} A_{4}-12 M^{2} B_{5}\right] \gamma^{\mu} p_{B} p_{N}+\left[A_{3}-4 B_{4}\right] \gamma^{\mu} \not p_{B} \not p_{N} \\
& +\left[2 A_{3}-16 B_{4}+48 C_{5}\right] p_{N}^{\mu} \not p_{N}+\left[16 C_{5}-4 B_{4}\right] p_{B}^{\mu} \not p_{B} \\
& \left.+\left[12 B_{4}-2 A_{3}-24 C_{5}\right] p_{B}^{\mu} \not p_{N}+\left[4 B_{4}-24 C_{5}\right] p_{N}^{\mu} \not p_{B}\right\} u\left(p_{N}\right) \epsilon_{\mu}^{*}\left(p_{B}\right) \\
= & \frac{i g^{\prime} g_{N}^{2}}{192 \pi^{2} M^{2}} \bar{u}\left(p_{N}-p_{B}\right) P_{L}\left\{\gamma^{\mu}\left(6 M^{2} \log \frac{\mu^{2}}{M^{2}}+p_{N}^{2}+3 p_{B}^{2}-3 p_{B} p_{N}+2 \not p_{B} \not p_{N}\right)\right. \\
& \left.+2 p_{N}^{\mu} \not p_{N}-2 p_{B}^{\mu} \not p_{B}-3 p_{B}^{\mu} \not p_{N}+p_{N}^{\mu} \not p_{B}\right\} u\left(p_{N}\right) \epsilon_{\mu}^{*}\left(p_{B}\right) .
\end{aligned}
$$

Here and in what follows $D \equiv k^{2}-M^{2}$. The second diagram leads to

$$
\begin{aligned}
i \mathcal{M}_{\mathrm{UV}}^{b}= & -g^{\prime} g_{N}^{2} \bar{u}\left(p_{N}-p_{B}\right) P_{L}\left\{\mu^{4-d} \int \frac{\mathrm{d}^{d} k}{(2 \pi)^{d}} \frac{1}{D^{3}}\left(\not k+\not p_{N}+M\right)\left(2 k^{\mu}+p_{B}^{\mu}\right)\right. \\
& \times\left[1-\frac{2 p_{N} k+p_{N}^{2}}{D}+\frac{4\left(k p_{N}\right)^{2}}{D^{2}}\right] \\
& \left.\times\left[1-\frac{2 p_{B} k+p_{B}^{2}}{D}+\frac{4\left(k p_{B}\right)^{2}}{D^{2}}\right]\right\} P_{R} u\left(p_{N}\right) \epsilon_{\mu}^{*}\left(p_{B}\right) \\
= & -g^{\prime} g_{N}^{2} \bar{u}\left(p_{N}-p_{B}\right) P_{L}\left\{2 \mu^{2 \epsilon} B_{3} \gamma^{\mu}+\left[8 C_{5}-2 B_{4}\right] \gamma^{\mu} p_{N}^{2}+\left[8 C_{5}-2 B_{4}\right] \gamma^{\mu} p_{B}^{2}\right. \\
& +8 C_{5} \gamma^{\mu} p_{B} p_{N}+\left[16 C_{5}-4 B_{4}\right] p_{N}^{\mu} \not_{N}+\left[16 C_{5}-2 B_{4}\right] p_{B}^{\mu} \not p_{B} \\
& \left.+\left[A_{3}-6 B_{4}+8 C_{5}\right] p_{B}^{\mu} \not p_{N}+8 C_{5} p_{N}^{\mu} \not p_{B}\right\} u\left(p_{N}\right) \epsilon_{\mu}^{*}\left(p_{B}\right) \\
= & \frac{i g^{\prime} g_{N}^{2}}{192 \pi^{2} M^{2}} \bar{u}\left(p_{N}-p_{B}\right) P_{L}\left\{\gamma^{\mu}\left(-6 M^{2} \log \frac{\mu^{2}}{M^{2}}-p_{N}^{2}-p_{B}^{2}+p_{B} p_{N}\right)\right. \\
& \left.-2 p_{N}^{\mu} \not_{N}+p_{B}^{\mu} \not \phi_{N}+p_{N}^{\mu} \not p_{B}\right\} u\left(p_{N}\right) \epsilon_{\mu}^{*}\left(p_{B}\right) .
\end{aligned}
$$


Adding the two pieces together:

$$
\begin{aligned}
i \mathcal{M}_{\mathrm{UV}}= & i \mathcal{M}_{\mathrm{UV}}^{a}+i \mathcal{M}_{\mathrm{UV}}^{b} \\
= & \frac{i g^{\prime} g_{N}^{2}}{96 \pi^{2} M^{2}} \bar{u}\left(p_{N}-p_{B}\right) P_{L}\left\{\gamma^{\mu}\left(p_{B}^{2}-p_{B} p_{N}+\not p_{B} \not p_{N}\right)\right. \\
& \left.-p_{B}^{\mu} \not p_{B}-p_{B}^{\mu} \not p_{N}+p_{N}^{\mu} \not \phi_{B}\right\} u\left(p_{N}\right) \epsilon_{\mu}^{*}\left(p_{B}\right) .
\end{aligned}
$$

\section{B.2 Amplitude for one Higgs and no gauge bosons}

This UV amplitude is represented by the diagram $(c)$ in figure 1. We have:

$$
\begin{aligned}
i \mathcal{M}_{\mathrm{UV}}= & -\frac{g_{N} g_{X} g_{L}}{\sqrt{2}} \bar{u}\left(p_{N}\right) P_{L}\left\{\mu^{4-d} \int \frac{\mathrm{d}^{d} k}{(2 \pi)^{d}} \frac{1}{D^{3}}\left(\not p_{N}+\not k+M\right)\left(\not p_{\nu}+\not k+M\right)\right. \\
& \times\left[1-\frac{2 k p_{N}+p_{N}^{2}}{D}+\frac{4\left(k p_{N}\right)^{2}}{D^{2}}\right] \\
& \left.\times\left[1-\frac{2 k p_{\nu}+p_{\nu}^{2}}{D}+\frac{4\left(k p_{\nu}\right)^{2}}{D^{2}}\right]\right\} P_{L} u\left(p_{\nu}\right) \\
= & -\frac{g_{N} g_{X} g_{L}}{\sqrt{2}} \bar{u}\left(p_{N}\right) P_{L}\left\{\mu^{2 \epsilon}(4-2 \epsilon) B_{3}+M^{2} A_{3}\right. \\
& -\left[6\left(B_{4}-4 C_{5}\right)+M^{2}\left(A_{4}-4 B_{5}\right)\right]\left(p_{\nu}^{2}+p_{N}^{2}\right) \\
& \left.+4\left(6 C_{5}+M^{2} B_{5}\right) p_{\nu} p_{N}+\left(A_{3}-4 B_{4}\right) \not p_{N} \not p_{\nu}\right\} u\left(p_{\nu}\right) \\
= & \frac{i g_{N} g_{X} g_{L}}{96 \sqrt{2} \pi^{2} M^{2}} \bar{u}\left(p_{N}\right) P_{L}\left\{6 M^{2}\left(1-\log \frac{\mu^{2}}{M^{2}}\right)-p_{\nu}^{2}-p_{N}^{2}+p_{\nu} p_{N}+\not p_{N} \not p_{\nu}\right\} u\left(p_{\nu}\right) .
\end{aligned}
$$

\section{B.3 Amplitude for one Higgs and one photon}

The relevant UV diagrams are depicted in figure 2. We have:

$$
\begin{aligned}
i \mathcal{M}_{\mathrm{UV}}^{a+b}= & \frac{g_{L} g_{X} g_{N} e}{\sqrt{2}} \bar{u}\left(p_{N}\right) P_{L} \int \frac{\mathrm{d}^{4} k}{(2 \pi)^{4}} \frac{1}{D^{4}}\left(\not p_{N}+\not k+M\right)\left[1-\frac{2 k p_{N}}{D}\right] \\
& \times\left\{\gamma^{\mu}\left(\not p_{\gamma}+\not p_{N}+\not k+M\right)\left[1-\frac{2 k\left(p_{\gamma}+p_{N}\right)}{D}\right]\right. \\
& \left.+\left(\not p_{h}+\not p_{N}+\not k+M\right) \gamma^{\mu}\left[1-\frac{2 k\left(p_{h}+p_{N}\right)}{D}\right]\right\} \\
& \times\left(\not p_{h}+\not p_{\gamma}+\not p_{N}+\not k+M\right)\left[1-\frac{2 k\left(p_{h}+p_{\gamma}+p_{N}\right)}{D}\right] \\
& \times P_{L} u\left(p_{\nu}\right) \epsilon_{\mu}^{*}\left(p_{\gamma}\right)
\end{aligned}
$$




$$
\begin{aligned}
= & \frac{g_{L} g_{X} g_{N} e}{\sqrt{2}} \bar{u}\left(p_{N}\right) P_{L}\left\{2\left[2 B_{4}-12 C_{5}+M^{2}\left(A_{4}-10 B_{5}\right)\right] p_{h}^{\mu}\right. \\
& +4\left[B_{4}-12 C_{5}-4 M^{2} B_{5}\right] p_{\gamma}^{\mu} \\
& +6\left[2 B_{4}-12 C_{5}+M^{2}\left(A_{4}-6 B_{5}\right)\right] p_{N}^{\mu} \\
& +\left[2 B_{4}-12 C_{5}+M^{2}\left(A_{4}+2 B_{5}\right)\right] \gamma^{\mu} \not p_{h} \\
& \left.+\left[2 B_{4}+12 C_{5}+M^{2}\left(3 A_{4}-2 B_{5}\right)\right] \gamma^{\mu} \not p_{\gamma}\right\} u\left(p_{\nu}\right) \epsilon_{\mu}^{*}\left(p_{\gamma}\right) \\
= & \frac{i g_{L} g_{X} g_{N} e}{96 \sqrt{2} \pi^{2} M^{2}} \bar{u}\left(p_{N}\right) P_{L}\left\{-p_{h}^{\mu}-p_{\gamma}^{\mu}+\gamma^{\mu} \not p_{h}+\gamma^{\mu} \not p_{\gamma}\right\} u\left(p_{\nu}\right) \epsilon_{\mu}^{*}\left(p_{\gamma}\right),
\end{aligned}
$$

and

$$
\begin{aligned}
i \mathcal{M}_{\mathrm{UV}}^{c}= & \frac{g_{L} g_{X} g_{N} e}{\sqrt{2}} \bar{u}\left(p_{N}\right) P_{L}\left\{\int \frac{\mathrm{d}^{4} k}{(2 \pi)^{4}} \frac{1}{D^{4}}\left(\not p_{\gamma}+\not p_{N}+\not k+M\right)\left(\not p_{h}+\not p_{\gamma}+\not p_{N}+\not k+M\right)\right. \\
& \times\left(p_{\gamma}^{\mu}+2 k^{\mu}\right)\left[1-\frac{2 k\left(p_{\gamma}+p_{N}\right)}{D}\right]\left[1-\frac{2 k\left(p_{h}+p_{\gamma}+p_{N}\right)}{D}\right] \\
& \left.\times\left[1-\frac{2 k p_{\gamma}}{D}\right]\right\} P_{L} u\left(p_{\nu}\right) \epsilon_{\mu}^{*}\left(p_{\gamma}\right) \\
= & \frac{g_{L} g_{X} g_{N} e}{\sqrt{2}} \bar{u}\left(p_{N}\right) P_{L}\left\{-2\left[12 C_{5}+2 M^{2} B_{5}\right] p_{h}^{\mu}+2 B_{4} \gamma^{\mu} \not p_{h}\right. \\
& +\left[8\left(B_{4}-9 C_{5}\right)+M^{2}\left(A_{4}-12 B_{5}\right)\right] p_{\gamma}^{\mu} \\
& \left.+4\left[B_{4}-12 C_{5}-2 M^{2} B_{5}\right] p_{N}^{\mu}\right\} u\left(p_{\nu}\right) \epsilon_{\mu}^{*}\left(p_{\gamma}\right) \\
= & \frac{i g_{L} g_{X} g_{N} e}{96 \sqrt{2} \pi^{2} M^{2}} \bar{u}\left(p_{N}\right) P_{L}\left\{p_{h}^{\mu}-\gamma^{\mu} \not p_{h}\right\} u\left(p_{\nu}\right) \epsilon_{\mu}^{*}\left(p_{\gamma}\right) .
\end{aligned}
$$

Adding eqs. (B.5) and (B.6) together, we get:

$$
i \mathcal{M}_{\mathrm{UV}}=i \mathcal{M}_{\mathrm{UV}}^{a+b}+i \mathcal{M}_{\mathrm{UV}}^{c}=\frac{i g_{L} g_{X} g_{N} e}{96 \sqrt{2} \pi^{2} M^{2}} \bar{u}\left(p_{N}\right) P_{L}\left\{\gamma^{\mu} \not p_{\gamma}-p_{\gamma}^{\mu}\right\} u\left(p_{\nu}\right) \epsilon_{\mu}^{*}\left(p_{\gamma}\right)
$$

\section{B.4 Amplitude for one Higgs and one $W$}

This amplitude in the UV is depicted by the diagram $(a)$ in figure 3 . We have:

$$
\begin{aligned}
i \mathcal{M}_{\mathrm{UV}}= & \frac{g_{N} g_{X} g_{L} g}{2} \bar{u}\left(p_{N}\right) P_{L}\left\{\int \frac{\mathrm{d}^{4} k}{(2 \pi)^{4}} \frac{1}{D^{4}}\left(\not p_{N}+\not k+M\right)\left(\not p_{h}+\not p_{N}+\not k+M\right) \gamma^{\mu}\right. \\
& \times\left(\not p_{W}+\not p_{h}+\not p_{N}+\not k+M\right)\left[1-\frac{2 k p_{N}}{D}\right] \\
& \left.\times\left[1-\frac{2 k\left(p_{h}+p_{N}\right)}{D}\right]\left[1-\frac{2 k\left(p_{W}+p_{h}+p_{N}\right)}{D}\right]\right\} \\
& \times P_{L} u\left(p_{e}\right) \epsilon_{\mu}^{*}\left(p_{W}\right)
\end{aligned}
$$




$$
\begin{aligned}
= & \frac{g_{N} g_{X} g_{L} g}{2} \bar{u}\left(p_{N}\right) P_{L}\left\{4 M^{2}\left(A_{4}-6 B_{5}\right) p_{N}^{\mu}+\left[4 B_{4}+2 M^{2}\left(A_{4}-8 B_{5}\right)\right] p_{h}^{\mu}\right. \\
& -8 M^{2} B_{5} p_{W}^{\mu}+\left[6\left(B_{4}-6 C_{5}\right)-M^{2}\left(A_{4}-6 B_{5}\right)\right] \gamma^{\mu} \not p_{N} \\
& +4\left(B_{4}-6 C_{5}+M^{2} B_{5}\right) \gamma^{\mu} \not p_{h} \\
& \left.+\left[4\left(B_{4}-3 C_{5}\right)+M^{2}\left(A_{4}+2 B_{5}\right)\right] \gamma^{\mu} \not p_{W}\right\} u\left(p_{e}\right) \epsilon_{\mu}^{*}\left(p_{W}\right) \\
= & \frac{i g_{N} g_{X} g_{L} g}{192 \pi^{2} M^{2}} \bar{u}\left(p_{N}\right) P_{L}\left\{p_{N}^{\mu}-2 p_{h}^{\mu}-p_{W}^{\mu}-\gamma^{\mu} \not p_{N}\right\} u\left(p_{e}\right) \epsilon_{\mu}^{*}\left(p_{W}\right) .
\end{aligned}
$$

\section{B.5 Amplitude for two Higgses and no gauge bosons}

This UV amplitude is given by the diagrams $(b)$ and $(c)$ in figure 3. Taking into account possible permutations of $p_{h}$ and $p_{h^{\prime}}$, we have:

$$
\begin{aligned}
i \mathcal{M}_{\mathrm{UV}}^{b}= & \frac{g_{N}^{2} g_{X}^{2}}{2} \bar{u}\left(p_{N^{\prime}}\right) P_{L} \int \frac{\mathrm{d}^{4} k}{(2 \pi)^{4}} \frac{1}{D^{4}}\left(\not p_{N^{\prime}}+\not k+M\right)\left[1-\frac{2 k p_{N^{\prime}}}{D}\right] \\
& \times\left\{\left(\not p_{N}-\not p_{h}+\not k+M\right)\left[1-\frac{2 k p_{N}}{D}+\frac{2 k p_{h}}{D}\right]\right. \\
& \left.+\left(\not p_{N}-\not p_{h^{\prime}}+\not k+M\right)\left[1-\frac{2 k p_{N}}{D}+\frac{2 k p_{h^{\prime}}}{D}\right]\right\} \\
& \times\left(\not p_{N}+\not k+M\right)\left[1-\frac{2 k p_{N}}{D}\right] P_{R} u\left(p_{N}\right) \\
= & \frac{g_{N}^{2} g_{X}^{2}}{2} \bar{u}\left(p_{N^{\prime}}\right) P_{L}\left\{2\left[2 B_{4}-24 C_{5}+2 M^{2} A_{4}-12 M^{2} B_{5}\right] \not p_{N}\right. \\
& +\left[2 B_{4}+12 C_{5}-M^{2} A_{4}+6 M^{2} B_{5}\right] \not p_{h} \\
& +\left[2 B_{4}+12 C_{5}-M^{2} A_{4}+6 M^{2} B_{5}\right] \not p_{h^{\prime}} \\
& \left.+2\left[4 B_{4}-12 C_{5}+M^{2} A_{4}-6 M^{2} B_{5}\right] \not p_{N^{\prime}}\right\} u\left(p_{N}\right) \\
= & \frac{i g_{N}^{2} g_{X}^{2}}{96 \pi^{2} M^{2}} \bar{u}\left(p_{N^{\prime}}\right) P_{L}\left\{\not p_{N}-\not p_{h}-\not p_{h^{\prime}}-\not p_{N^{\prime}}\right\} u\left(p_{N}\right)=0,
\end{aligned}
$$

by virtue of the momentum conservation. Thus, we get:

$$
\begin{aligned}
i \mathcal{M}_{\mathrm{UV}}= & i \mathcal{M}_{\mathrm{UV}}^{c}=g_{N}^{2} \lambda_{\varphi H} \bar{u}\left(p_{N^{\prime}}\right) P_{L}\left\{\int \frac{\mathrm{d}^{4} k}{(2 \pi)^{4}} \frac{1}{D^{3}}\left(\not p_{N}+\not k+M\right)\right. \\
& \left.\times\left[1-\frac{2 k p_{N}}{D}\right]\left[1-\frac{2 k\left(p_{N}-p_{N^{\prime}}\right)}{D}\right]\right\} P_{R} u\left(p_{N}\right) \\
= & g_{N}^{2} \lambda_{\varphi H} \bar{u}\left(p_{N^{\prime}}\right) P_{L}\left\{\left[A_{3}-4 B_{4}\right] \not p_{N}+2 B_{4} \not p_{N^{\prime}}\right\} u\left(p_{N}\right) \\
= & -\frac{i g_{N}^{2} \lambda_{\varphi H}}{96 \pi^{2} M^{2}} \bar{u}\left(p_{N^{\prime}}\right) P_{L}\left\{\not p_{N}+\not p_{N^{\prime}}\right\} u\left(p_{N}\right) .
\end{aligned}
$$




\section{B.6 Amplitude for two Higgses and one $W^{3}$}

The relevant diagram in the UV is the diagram $(a)$ in figure 4 . We have:

$$
\begin{aligned}
i \mathcal{M}_{\mathrm{UV}} & =-\frac{g g_{N}^{2} g_{X}^{2}}{2} \bar{u}\left(p_{N^{\prime}}\right) P_{L} \int \frac{\mathrm{d}^{4} k}{(2 \pi)^{4}} \frac{1}{D^{5}}(\not k+M)^{2} \gamma^{\mu}(\not k+M)^{2} P_{R} u\left(p_{N}\right) \epsilon_{\mu}^{*}\left(p_{W^{3}}\right) \\
& =-\frac{g g_{N}^{2} g_{X}^{2}}{2} \bar{u}\left(p_{N^{\prime}}\right) P_{L}\left(24 C_{5}+M^{4} A_{5}\right) \gamma^{\mu} u\left(p_{N}\right) \epsilon_{\mu}^{*}\left(p_{W^{3}}\right) \\
& =\frac{i g g_{N}^{2} g_{X}^{2}}{96 \pi^{2} M^{2}} \bar{u}\left(p_{N^{\prime}}\right) P_{L} \gamma^{\mu} u\left(p_{N}\right) \epsilon_{\mu}^{*}\left(p_{W^{3}}\right) .
\end{aligned}
$$

\section{B.7 Amplitude for three Higgses and no gauge bosons}

This UV amplitude is represented by the diagrams $(b)$ and $(c)$ in figure 4 . We have:

$$
\begin{aligned}
i \mathcal{M}_{\mathrm{UV}}^{b} & =-\frac{3 g_{N} g_{X}^{3} g_{L}}{\sqrt{2}} \bar{u}\left(p_{N}\right) P_{L} \int \frac{\mathrm{d}^{4} k}{(2 \pi)^{4}} \frac{1}{D^{5}}(\not k+M)^{4} P_{L} u\left(p_{\nu}\right) \\
& =-\frac{3 g_{N} g_{X}^{3} g_{L}}{\sqrt{2}} \bar{u}\left(p_{N}\right) P_{L}\left(24 C_{5}+24 M^{2} B_{5}+M^{4} A_{5}\right) u\left(p_{\nu}\right) \\
& =-\frac{i g_{N} g_{X}^{3} g_{L}}{32 \sqrt{2} \pi^{2} M^{2}} \bar{u}\left(p_{N}\right) P_{L} u\left(p_{\nu}\right), \\
i \mathcal{M}_{\mathrm{UV}}^{c} & =-\frac{3 \lambda_{\varphi H} g_{N} g_{X} g_{L}}{\sqrt{2}} \bar{u}\left(p_{N}\right) P_{L} \int \frac{\mathrm{d}^{4} k}{(2 \pi)^{4}} \frac{1}{D^{4}}(\not k+M)^{2} P_{L} u\left(p_{\nu}\right) \\
& =-\frac{3 \lambda_{\varphi H} g_{N} g_{X} g_{L}}{\sqrt{2}} \bar{u}\left(p_{N}\right) P_{L}\left(4 B_{4}+M^{2} A_{4}\right) u\left(p_{\nu}\right) \\
& =\frac{i \lambda_{\varphi H} g_{N} g_{X} g_{L}}{32 \sqrt{2} \pi^{2} M^{2}} \bar{u}\left(p_{N}\right) P_{L} u\left(p_{\nu}\right) .
\end{aligned}
$$

Finally,

$$
i \mathcal{M}_{\mathrm{UV}}=i \mathcal{M}_{\mathrm{UV}}^{b}+i \mathcal{M}_{\mathrm{UV}}^{c}=\frac{i g_{N} g_{X} g_{L}}{32 \sqrt{2} \pi^{2} M^{2}}\left(\lambda_{\varphi H}-g_{X}^{2}\right) \bar{u}\left(p_{N}\right) P_{L} u\left(p_{\nu}\right) .
$$

\section{B.8 Amplitude for four $N$ fermions}

This UV amplitude is depicted by the diagram $(a)$ in figure 5 (note that there is a second diagram with opposite sign due to the exchange of identical fermions). We have:

$$
\begin{aligned}
i \mathcal{M}_{\mathrm{UV}}= & g_{N}^{4} \int \frac{\mathrm{d}^{4} k}{(2 \pi)^{4}} \frac{1}{D^{4}}\left\{\left[\bar{u}\left(p_{3}\right) P_{L}(\not k+M) P_{R} u\left(p_{1}\right)\right]\left[\bar{u}\left(p_{4}\right) P_{L}(\not k+M) P_{R} u\left(p_{2}\right)\right]\right. \\
& \left.-\left[\bar{u}\left(p_{4}\right)(\not k+M) u\left(p_{1}\right)\right]\left[\bar{u}\left(p_{3}\right)(\not k+M) P_{R} u\left(p_{2}\right)\right]\right\} \\
= & g_{N}^{4} B_{4}\left\{\left[\bar{u}\left(p_{3}\right) \gamma^{\mu} P_{R} u\left(p_{1}\right)\right]\left[\bar{u}\left(p_{4}\right) \gamma_{\mu} P_{R} u\left(p_{2}\right)\right]\right. \\
& \left.-\left[\bar{u}\left(p_{4}\right) \gamma^{\mu} P_{R} u\left(p_{1}\right)\right]\left[\bar{u}\left(p_{3}\right) \gamma_{\mu} P_{R} u\left(p_{2}\right)\right]\right\} \\
= & 2 g_{N}^{4} B_{4}\left[\bar{u}\left(p_{3}\right) \gamma^{\mu} P_{R} u\left(p_{1}\right)\right]\left[\bar{u}\left(p_{4}\right) \gamma_{\mu} P_{R} u\left(p_{2}\right)\right] \\
= & -\frac{i g_{N}^{4}}{96 \pi^{2} M^{2}}\left[\bar{u}\left(p_{3}\right) \gamma^{\mu} P_{R} u\left(p_{1}\right)\right]\left[\bar{u}\left(p_{4}\right) \gamma_{\mu} P_{R} u\left(p_{2}\right)\right] .
\end{aligned}
$$

In the penultimate step, we have rearranged the spinors using a Fierz identity. 
(a)

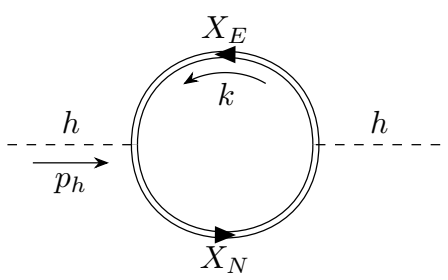

(b)

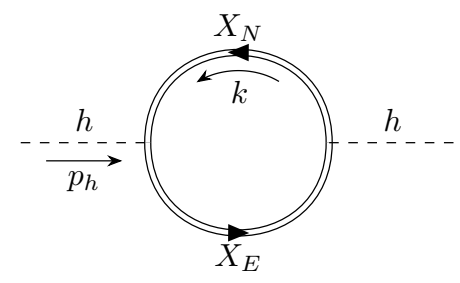

(c)

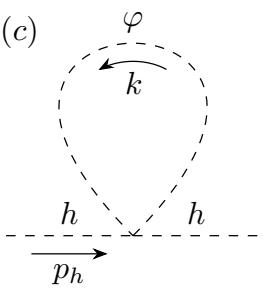

Figure 7. Diagrams contributing to the Higgs self-energy in the UV.

\section{B.9 Amplitude for two $N$ fermions and two neutrinos}

This amplitude in the UV is given by the diagram (b) in figure 5 . We have:

$$
\begin{aligned}
i \mathcal{M}_{\mathrm{UV}} & =g_{N}^{2} g_{L}^{2} \int \frac{\mathrm{d}^{4} k}{(2 \pi)^{4}} \frac{1}{D^{4}}\left[\bar{u}\left(p_{\nu^{\prime}}\right) P_{R}(\not k+M) P_{L} u\left(p_{\nu}\right)\right]\left[\bar{u}\left(p_{N^{\prime}}\right) P_{L}(\not k+M) P_{R} u\left(p_{N}\right)\right] \\
& =g_{N}^{2} g_{L}^{2} B_{4}\left[\bar{u}\left(p_{\nu^{\prime}}\right) \gamma^{\mu} P_{L} u\left(p_{\nu}\right)\right]\left[\bar{u}\left(p_{N^{\prime}}\right) \gamma_{\mu} P_{R} u\left(p_{N}\right)\right] \\
& =-\frac{i g_{N}^{2} g_{L}^{2}}{192 \pi^{2} M^{2}}\left[\bar{u}\left(p_{\nu^{\prime}}\right) \gamma^{\mu} P_{L} u\left(p_{\nu}\right)\right]\left[\bar{u}\left(p_{N^{\prime}}\right) \gamma_{\mu} P_{R} u\left(p_{N}\right)\right]
\end{aligned}
$$

\section{Matching of renormalisable terms}

Corrections to the Higgs propagator. The diagrams $(a)$ and $(b)$ in figure 7 lead to the following contribution to the Higgs self-energy:

$$
\begin{aligned}
-i\left(M_{\mathrm{UV}}^{2}\right)^{a+b}= & -g_{X}^{2} \mu^{4-d} \int \frac{\mathrm{d}^{d} k}{(2 \pi)^{d}} \frac{1}{D^{2}}\left\{\operatorname{tr}\left[\left(\not k+\not p_{h}+M\right)(\not k+M)\right]\right. \\
& \left.\times\left[1-\frac{2 k p_{h}+p_{h}^{2}}{D}+\frac{4\left(k p_{h}\right)^{2}}{D^{2}}\right]\right\} \\
= & -4 g_{X}^{2} \mu^{2 \epsilon}\left\{(4-2 \epsilon) B_{2}+M^{2} A_{2}\right. \\
& \left.+\left[(6-2 \epsilon)\left(4 C_{4}-B_{3}\right)+M^{2}\left(4 B_{4}-A_{3}\right)\right] p_{h}^{2}\right\} \\
= & -\frac{i g_{X}^{2}}{12 \pi^{2}}\left\{3\left(1+3 \log \frac{\mu^{2}}{M^{2}}\right) M^{2}+\left(1-3 \log \frac{\mu^{2}}{M^{2}}\right) p_{h}^{2}\right\},
\end{aligned}
$$

The diagram $(c)$ in figure 7 gives

$$
-i\left(M_{\mathrm{UV}}^{2}\right)^{c}=\lambda_{\varphi H} \mu^{4-d} \int \frac{\mathrm{d}^{d} k}{(2 \pi)^{d}} \frac{1}{D}=\lambda_{\varphi H} \mu^{2 \epsilon} A_{1}=\frac{i \lambda_{\varphi H}}{16 \pi^{2}}\left(1+\log \frac{\mu^{2}}{M^{2}}\right) M^{2} .
$$




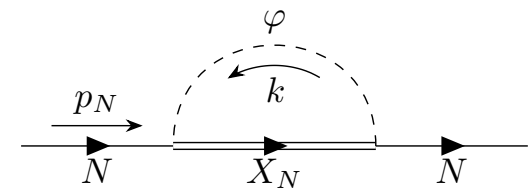

(a)

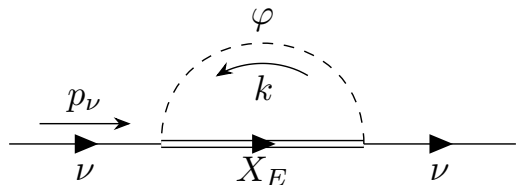

(b)

Figure 8. Diagrams contributing to the $N$ and $\nu$ self-energies in the UV.

Finally, their sum yields

$$
\begin{aligned}
-i M_{\mathrm{UV}}^{2}=-i\left(M_{\mathrm{UV}}^{2}\right)^{a+b}-i\left(M_{\mathrm{UV}}^{2}\right)^{c}= & -\frac{i}{48 \pi^{2}}\left[3\left(4 g_{X}^{2}-\lambda_{\varphi H}\right) M^{2}+4 g_{X}^{2} p_{h}^{2}\right] \\
& -\frac{i}{16 \pi^{2}}\left[\left(12 g_{X}^{2}-\lambda_{\varphi H}\right) M^{2}-4 g_{X}^{2} p_{h}^{2}\right] \log \frac{\mu^{2}}{M^{2}}
\end{aligned}
$$

Corrections to the fermion propagators. For the contribution to the self-energy of $N$ depicted by the diagram (a) in figure 8 , we find

$$
\begin{aligned}
-i \Sigma_{\mathrm{UV}} & =g_{N}^{2} P_{L} \mu^{4-d} \int \frac{\mathrm{d}^{d} k}{(2 \pi)^{d}} \frac{1}{D^{2}}\left(\not k+\not p_{N}+M\right)\left[1-\frac{2 k p_{N}}{D}\right] P_{R} \\
& =g_{N}^{2} \mu^{2 \epsilon}\left(A_{2}-2 B_{3}\right) P_{L} \not p_{N}=\frac{i g_{N}^{2}}{32 \pi^{2}} \log \frac{\mu^{2}}{M^{2}} P_{L} \not p_{N} .
\end{aligned}
$$

A similar contribution to the neutrino self-energy represented by the diagram $(b)$ in figure 8 reads

$$
\begin{aligned}
-i \Sigma_{\mathrm{UV}} & =g_{L}^{2} P_{R} \mu^{4-d} \int \frac{\mathrm{d}^{d} k}{(2 \pi)^{d}} \frac{1}{D^{2}}\left(\not k+\not \nu_{\nu}+M\right)\left[1-\frac{2 k p_{\nu}}{D}\right] P_{L} \\
& =g_{L}^{2} \mu^{2 \epsilon}\left(A_{2}-2 B_{3}\right) P_{R} \not \phi_{\nu}=\frac{i g_{L}^{2}}{32 \pi^{2}} \log \frac{\mu^{2}}{M^{2}} P_{R} \not \phi_{\nu} .
\end{aligned}
$$

Corrections to the gauge boson propagators. There are four diagrams contributing to the self-energy of $B_{\mu}$, see figure 9 . The diagram $(a)$ gives

$$
\begin{aligned}
i\left(\Pi_{\mathrm{UV}}^{\mu \nu}\right)^{a} & =g^{\prime 2} \mu^{4-d} \int \frac{\mathrm{d}^{d} k}{(2 \pi)^{d}} \frac{1}{D^{2}}\left(2 k^{\mu}+p_{B}^{\mu}\right)\left(2 k^{\nu}+p_{B}^{\nu}\right)\left[1-\frac{2 k p_{B}+p_{B}^{2}}{D}+\frac{4\left(k p_{B}\right)^{2}}{D^{2}}\right] \\
& =g^{\prime 2} \mu^{2 \epsilon}\left[4 B_{2} g^{\mu \nu}+\left(16 C_{4}-4 B_{3}\right) p_{B}^{2} g^{\mu \nu}+\left(A_{2}-8 B_{3}+32 C_{4}\right) p_{B}^{\mu} p_{B}^{\nu}\right] \\
& =\frac{i g^{\prime 2}}{48 \pi^{2}}\left[6 M^{2}\left(1+\log \frac{\mu^{2}}{M^{2}}\right) g^{\mu \nu}-\log \frac{\mu^{2}}{M^{2}}\left(p_{B}^{2} g^{\mu \nu}-p_{B}^{\mu} p_{B}^{\nu}\right)\right] .
\end{aligned}
$$

The diagram (b) yields

$$
\begin{aligned}
i\left(\Pi_{\mathrm{UV}}^{\mu \nu}\right)^{b} & =-2 g^{\prime 2} g^{\mu \nu} \mu^{4-d} \int \frac{\mathrm{d}^{d} k}{(2 \pi)^{d}} \frac{1}{D} \\
& =-2 g^{\prime 2} g^{\mu \nu} \mu^{2 \epsilon} A_{1}=-\frac{i g^{\prime 2}}{8 \pi^{2}} M^{2}\left(1+\log \frac{\mu^{2}}{M^{2}}\right) g^{\mu \nu} .
\end{aligned}
$$



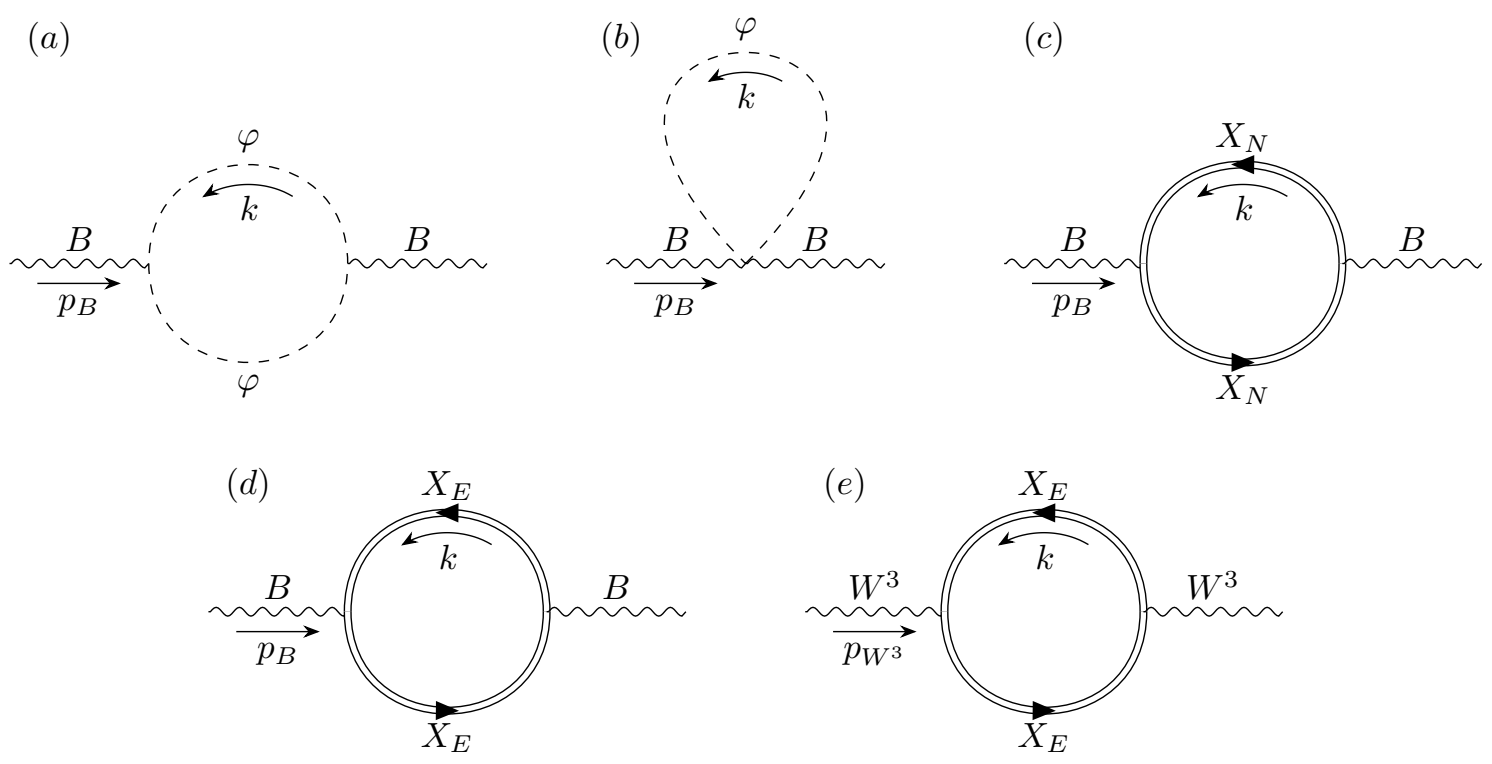

Figure 9. Diagrams contributing to the $B$ and $W^{3}$ self-energies in the UV.

Further, for the diagram $(c)$ we find

$$
\begin{aligned}
i\left(\Pi_{\mathrm{UV}}^{\mu \nu}\right)^{c}= & -g^{\prime 2} \mu^{4-d} \int \frac{\mathrm{d}^{d} k}{(2 \pi)^{d}}\left\{\frac{1}{D^{2}} \operatorname{tr}\left[\gamma^{\nu}\left(\not k+\not p_{B}+M\right) \gamma^{\mu}(\not k+M)\right]\right. \\
& \left.\times\left[1-\frac{2 k p_{B}+p_{B}^{2}}{D}+\frac{4\left(k p_{B}\right)^{2}}{D^{2}}\right]\right\} \\
= & -4 g^{\prime 2} \mu^{2 \epsilon}\left\{\left[M^{2} A_{2}+(2 \epsilon-2) B_{2}\right] g^{\mu \nu}+\left[16 C_{4}-4 B_{3}\right] p_{B}^{\mu} p_{B}^{\nu}\right. \\
& \left.+\left[(4-2 \epsilon)\left(B_{3}-4 C_{4}\right)-M^{2}\left(A_{3}-4 B_{4}\right)\right] p_{B}^{2} g^{\mu \nu}\right\} \\
= & -\frac{i g^{\prime 2}}{12 \pi^{2}} \log \frac{\mu^{2}}{M^{2}}\left(p_{B}^{2} g^{\mu \nu}-p_{B}^{\mu} p_{B}^{\nu}\right) .
\end{aligned}
$$

Finally, the diagram $(d)$ leads to the same result divided by 4 because of $Y_{X_{E}}=1 / 2$ and multiplied by 2 because both $X_{E}^{+}$and $X_{E}^{0}$ contribute. Summing all contributions we obtain

$$
i \Pi_{\mathrm{UV}}^{\mu \nu}=-\frac{7 i g^{\prime 2}}{48 \pi^{2}} \log \frac{\mu^{2}}{M^{2}}\left(p_{B}^{2} g^{\mu \nu}-p_{B}^{\mu} p_{B}^{\nu}\right)
$$

Computation of the diagram (e) in figure 9 providing a contribution to the $W_{\mu}^{3}$ selfenergy is almost the same as that in eq. (C.8). As a result, we find

$$
i \Pi_{\mathrm{UV}}^{\mu \nu}=-\frac{i g^{2}}{24 \pi^{2}} \log \frac{\mu^{2}}{M^{2}}\left(p_{W^{3}}^{2} g^{\mu \nu}-p_{W^{3}}^{\mu} p_{W^{3}}^{\nu}\right)
$$




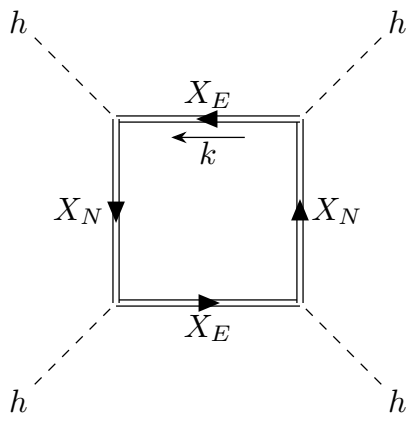

(a)

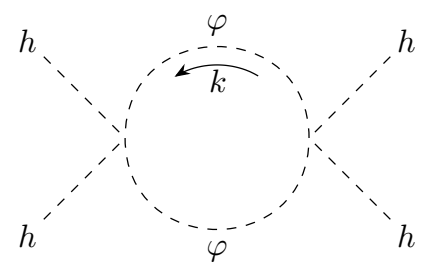

(b)

Figure 10. Diagrams contributing to the amplitude $\langle h h h h\rangle$ in the UV.

Corrections to the Higgs quartic coupling. The diagram $(a)$ in figure 10 reads

$$
\begin{aligned}
i \mathcal{M}_{\mathrm{UV}}^{a} & =-12 \frac{g_{X}^{4}}{4} \mu^{4-d} \int \frac{\mathrm{d}^{d} k}{(2 \pi)^{d}} \frac{\operatorname{tr}\left[(\not k+M)^{4}\right]}{D^{4}} \\
& =-3 g_{X}^{4}\left[M^{4} A_{4}+24 M^{2} B_{4}+\mu^{2 \epsilon}(4-2 \epsilon)(6-2 \epsilon) C_{4}\right] \\
& =\frac{2 i g_{X}^{4}}{\pi^{2}}\left(1-\frac{3}{8} \log \frac{\mu^{2}}{M^{2}}\right) .
\end{aligned}
$$

The diagram $(b)$ in figure 10 gives

$$
i \mathcal{M}_{\mathrm{UV}}^{b}=3 \lambda_{\varphi H}^{2} \mu^{4-d} \int \frac{\mathrm{d}^{d} k}{(2 \pi)^{d}} \frac{1}{D^{2}}=3 \lambda_{\varphi H}^{2} \mu^{2 \epsilon} A_{2}=\frac{3 i \lambda_{\varphi H}^{2}}{16 \pi^{2}} \log \frac{\mu^{2}}{M^{2}} .
$$

Finally,

$$
i \mathcal{M}_{\mathrm{UV}}=i \mathcal{M}_{\mathrm{UV}}^{a}+i \mathcal{M}_{\mathrm{UV}}^{b}=\frac{2 i g_{X}^{4}}{\pi^{2}}+\frac{i}{16 \pi^{2}}\left(3 \lambda_{\varphi H}^{2}-12 g_{X}^{4}\right) \log \frac{\mu^{2}}{M^{2}} .
$$

Final remarks. In light of the previous computations, we see that at the matching scale $\mu=M$, most of the one-loop corrections to renormalisable IR parameters vanish. The only exceptions are the Higgs mass parameter, kinetic term and quartic coupling. The relevant part of the IR Lagrangian reads

$$
\mathcal{L}_{\mathrm{SM}+N}^{\mathrm{IR}} \supset \alpha_{H}^{\mathrm{IR}}\left(D_{\mu} H\right)^{\dagger}\left(D^{\mu} H\right)+\left(\mu_{H}^{\mathrm{IR}}\right)^{2} H^{\dagger} H-\frac{1}{2} \lambda_{H}^{\mathrm{IR}}\left(H^{\dagger} H\right)^{2} .
$$

(Note that in the UV the Higgs field is canonically normalised and therefore $\alpha_{H}^{\mathrm{UV}}=1$.) Thus, the matching conditions read:

$$
i \alpha_{H}^{\mathrm{IR}} p_{h}^{2}+i\left(\mu_{H}^{\mathrm{IR}}\right)^{2}=i p_{h}^{2}+i\left(\mu_{H}^{\mathrm{UV}}\right)^{2}-i M_{\mathrm{UV}}^{2},
$$

where $-i M_{\mathrm{UV}}^{2}$ is given in eq. (C.3) and

$$
-3 i \lambda_{H}^{\mathrm{IR}}=-3 i \lambda_{H}^{\mathrm{UV}}+i \mathcal{M}_{\mathrm{UV}},
$$

with $i \mathcal{M}_{\mathrm{UV}}$ from eq. (C.13). At the matching scale $\mu=M$ they lead to

$$
\alpha_{H}^{\mathrm{IR}}=1-\frac{g_{X}^{2}}{12 \pi^{2}}, \quad\left(\mu_{H}^{\mathrm{IR}}\right)^{2}=\left(\mu_{H}^{\mathrm{UV}}\right)^{2}+\frac{\lambda_{\varphi H}-4 g_{X}^{2}}{16 \pi^{2}} M^{2}, \quad \lambda_{H}^{\mathrm{IR}}=\lambda_{H}^{\mathrm{UV}}-\frac{2 g_{X}^{4}}{3 \pi^{2}} .
$$




\begin{tabular}{|c|c|c|}
\hline Dipole & \multicolumn{2}{|c|}{$\mathcal{O}_{N \gamma}=\overline{\nu_{L}} \sigma^{\mu \nu} N A_{\mu \nu}$} \\
\hline \multirow{3}{*}{ 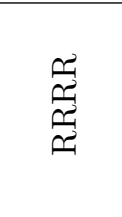 } & \multicolumn{2}{|c|}{$\mathcal{O}_{N N}^{V, R R}=\left(\bar{N} \gamma_{\mu} N\right)\left(\bar{N} \gamma^{\mu} N\right)$} \\
\hline & $\mathcal{O}_{e N}^{V, R R}=\left(\overline{e_{R}} \gamma_{\mu} e_{R}\right)\left(\bar{N} \gamma^{\mu} N\right)$ & $\mathcal{O}_{u N}^{V, R R}=\left(\overline{u_{R}} \gamma_{\mu} u_{R}\right)\left(\bar{N} \gamma^{\mu} N\right)$ \\
\hline & $\mathcal{O}_{d N}^{V, R R}=\left(\overline{d_{R}} \gamma_{\mu} d_{R}\right)\left(\bar{N} \gamma^{\mu} N\right)$ & $\mathcal{O}_{u d e N}^{V, R R}=\left(\overline{u_{R}} \gamma_{\mu} d_{R}\right)\left(\overline{e_{R}} \gamma^{\mu} N\right)$ \\
\hline \multirow{3}{*}{ 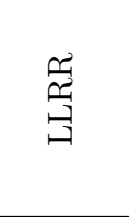 } & $\mathcal{O}_{\nu N}^{V, L R}=\left(\overline{\nu_{L}} \gamma_{\mu} \nu_{L}\right)\left(\bar{N} \gamma^{\mu} N\right)$ & $\mathcal{O}_{e N}^{V, L R}=\left(\overline{e_{L}} \gamma_{\mu} e_{L}\right)\left(\bar{N} \gamma^{\mu} N\right)$ \\
\hline & $\mathcal{O}_{u N}^{V, L R}=\left(\overline{u_{L}} \gamma_{\mu} u_{L}\right)\left(\bar{N} \gamma^{\mu} N\right)$ & $\mathcal{O}_{d N}^{V, L R}=\left(\overline{d_{L}} \gamma_{\mu} d_{L}\right)\left(\bar{N} \gamma^{\mu} N\right)$ \\
\hline & \multicolumn{2}{|c|}{$\mathcal{O}_{u d e N}^{V, L R}=\left(\overline{u_{L}} \gamma_{\mu} d_{L}\right)\left(\overline{e_{R}} \gamma^{\mu} N\right)$} \\
\hline \multirow{5}{*}{ 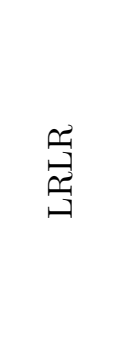 } & \multicolumn{2}{|c|}{$\mathcal{O}_{N N}^{S, R R}=\left(\overline{\nu_{L}} N\right)\left(\overline{\nu_{L}} N\right)$} \\
\hline & $\mathcal{O}_{e N}^{S, R R}=\left(\overline{e_{L}} e_{R}\right)\left(\overline{\nu_{L}} N\right)$ & $\mathcal{O}_{e N}^{T, R R}=\left(\overline{e_{L}} \sigma_{\mu \nu} e_{R}\right)\left(\overline{\nu_{L}} \sigma^{\mu \nu} N\right)$ \\
\hline & $\mathcal{O}_{u N}^{S, R R}=\left(\overline{u_{L}} u_{R}\right)\left(\overline{\nu_{L}} N\right)$ & $\mathcal{O}_{u N}^{T, R R}=\left(\overline{u_{L}} \sigma_{\mu \nu} u_{R}\right)\left(\overline{\nu_{L}} \sigma^{\mu \nu} N\right)$ \\
\hline & $\mathcal{O}_{d N}^{S, R R}=\left(\overline{d_{L}} d_{R}\right)\left(\overline{\nu_{L}} N\right)$ & $\mathcal{O}_{d N}^{T, R R}=\left(\overline{d_{L}} \sigma_{\mu \nu} d_{R}\right)\left(\overline{\nu_{L}} \sigma^{\mu \nu} N\right)$ \\
\hline & $\mathcal{O}_{u d e N}^{S, R R}=\left(\overline{u_{L}} d_{R}\right)\left(\overline{e_{L}} N\right)$ & $\mathcal{O}_{u d e N}^{T, R R}=\left(\overline{u_{L}} \sigma_{\mu \nu} d_{R}\right)\left(\overline{e_{L}} \sigma^{\mu \nu} N\right)$ \\
\hline \multirow{2}{*}{ 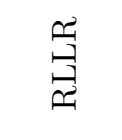 } & $\mathcal{O}_{e N}^{S, L R}=\left(\overline{e_{R}} e_{L}\right)\left(\overline{\nu_{L}} N\right)$ & $\mathcal{O}_{u N}^{S, L R}=\left(\overline{u_{R}} u_{L}\right)\left(\overline{\nu_{L}} N\right)$ \\
\hline & $\mathcal{O}_{d N}^{S, L R}=\left(\overline{d_{R}} d_{L}\right)\left(\overline{\nu_{L}} N\right)$ & $\mathcal{O}_{u d e N}^{S, L R}=\left(\overline{u_{R}} d_{L}\right)\left(\overline{e_{L}} N\right)$ \\
\hline
\end{tabular}

Table 3. List of $\nu$ LEFT lepton number conserving operators involving $N$. The addition of h.c. is implied when needed. Note that the operators obtained from the non-Hermitian operators in the table with multiplication by the imaginary unit are also present. The notation follows that of ref. [17], although we have not tried to minimise the number of operators involving $\sigma_{\mu \nu}$.

\section{Matching the $\nu$ SMEFT onto the $\nu$ LEFT and anomalous dimensions}

The full list of lepton number conserving dimension-six operators in the $\nu$ LEFT involving $N$ is shown in table 3. (Those not involving $N$ can be found in ref. [17].) The following relations hold at the EW matching scale (note that we are ignoring family indices):

$$
\begin{aligned}
& \frac{\alpha_{N \gamma}}{v}=\frac{v}{\sqrt{2} \Lambda^{2}}\left(\alpha_{N B} c_{W}+\alpha_{N W} s_{W}\right), \quad(\text { D.1 }) \quad \frac{\alpha_{N N}^{V, R R}}{v^{2}}=\frac{\alpha_{N N}}{\Lambda^{2}}, \\
& \frac{\alpha_{e N}^{V, R R}}{v^{2}}=\frac{\alpha_{e N}}{\Lambda^{2}}-\frac{g_{Z}^{2} Z_{e_{R}} Z_{N}}{m_{Z}^{2}}, \\
& \frac{\alpha_{d N}^{V, R R}}{v^{2}}=\frac{\alpha_{d N}}{\Lambda^{2}}-\frac{g_{Z}^{2} Z_{d_{R}} Z_{N}}{m_{Z}^{2}}, \\
& \frac{\alpha_{\nu N}^{V, L R}}{v^{2}}=\frac{\alpha_{L N}}{\Lambda^{2}}-\frac{g_{Z}^{2} Z_{\nu_{L}} Z_{N}}{m_{Z}^{2}}, \\
& \frac{\alpha_{u N}^{V, L R}}{v^{2}}=\frac{\alpha_{Q N}}{\Lambda^{2}}-\frac{g_{Z}^{2} Z_{u_{L}} Z_{N}}{m_{Z}^{2}}, \\
& \frac{\alpha_{u N}^{V, R R}}{v^{2}}=\frac{\alpha_{u N}}{\Lambda^{2}}-\frac{g_{Z}^{2} Z_{u_{R}} Z_{N}}{m_{Z}^{2}}, \\
& \frac{\alpha_{u d e N}^{V, R R}}{v^{2}}=\frac{\alpha_{d u N e}}{\Lambda^{2}}, \\
& \frac{\alpha_{e N}^{V, L R}}{v^{2}}=\frac{\alpha_{L N}}{\Lambda^{2}}-\frac{g_{Z}^{2} Z_{e_{L}} Z_{N}}{m_{Z}^{2}}, \\
& \frac{\alpha_{d N}^{V, L R}}{v^{2}}=\frac{\alpha_{Q N}}{\Lambda^{2}}-\frac{g_{Z}^{2} Z_{d_{L}} Z_{N}}{m_{Z}^{2}},
\end{aligned}
$$




$$
\begin{aligned}
\frac{\alpha_{u d e N}^{V, L R}}{v^{2}} & =-\frac{g^{2} W_{N}}{2 m_{W}^{2}}, \\
\frac{\alpha_{e N}^{S, R R}}{v^{2}} & =\frac{3 \alpha_{L N L e}}{2 \Lambda^{2}}, \\
\alpha_{u N}^{S, R R} & =0 \\
\frac{\alpha_{d N}^{S, R R}}{v^{2}} & =\frac{\alpha_{L N Q d}}{\Lambda^{2}}-\frac{\alpha_{L d Q N}}{2 \Lambda^{2}}, \\
\frac{\alpha_{u d e N}^{S, R R}}{v^{2}} & =\frac{\alpha_{L d Q N}}{2 \Lambda^{2}}-\frac{\alpha_{L N Q d}}{\Lambda^{2}}, \\
\frac{\alpha_{e N}^{S, L R}}{v^{2}} & =\frac{g^{2} W_{N}}{m_{W}^{2}}, \\
\alpha_{d N}^{S, L R} & =0
\end{aligned}
$$

$$
\alpha_{N N}^{S, R R}=0
$$$$
\frac{\alpha_{e N}^{T, R R}}{v^{2}}=\frac{\alpha_{L N L e}}{8 \Lambda^{2}},
$$

$$
\alpha_{u N}^{T, R R}=0,
$$$$
\frac{\alpha_{d N}^{T, R R}}{v^{2}}=-\frac{\alpha_{L d Q N}}{8 \Lambda^{2}}
$$

$$
\begin{aligned}
& \frac{\alpha_{u d e N}^{T, R R}}{v^{2}}=\frac{\alpha_{L d Q N}}{8 \Lambda^{2}}, \\
& \frac{\alpha_{u N}^{S, L R}}{v^{2}}=\frac{\alpha_{Q u N L}}{\Lambda^{2}},
\end{aligned}
$$$$
\frac{\alpha_{u d e N}^{S, L R}}{v^{2}}=\frac{\alpha_{Q u N L}}{\Lambda^{2}} .
$$

The coupling $g_{Z}$ is defined as $g_{Z}=e /\left(s_{W} c_{W}\right)$. We have also defined $Z_{\psi_{\mathrm{SM}}}=T_{3}-Q s_{W}^{2}$ and $Z_{N}=-\alpha_{H N} v^{2} /\left(2 \Lambda^{2}\right)$ as well as $W_{N}=\alpha_{H N e} v^{2} /\left(2 \Lambda^{2}\right)$. Note that we can neglect EFT effects in the non $N$ fermion couplings to the $Z$ and $W$ because they would lead to dimension-eight contributions.

In our case, the only operators that are generated are the dipole as well as vector type $\mathrm{RR}$ and LR four-fermions with two Ns. They renormalise due to quantum corrections depicted by the diagrams in figure 11 . Using the notation

$$
\dot{\alpha} \equiv 16 \pi^{2} \mu \frac{\mathrm{d} \alpha}{\mathrm{d} \mu},
$$

we obtain:

$$
\begin{aligned}
\dot{\alpha}_{N \gamma}= & \frac{4}{3}\left(3 q_{e}^{2}+3 N_{c} q_{d}^{2}+2 N_{c} q_{u}^{2}\right) e^{2} \alpha_{N \gamma}, \\
\dot{\alpha}_{\psi N}^{V, R R}= & \frac{4}{3} e^{2} q_{\psi}\left[N_{c} q_{u}\left(\alpha_{u N}^{V, R R}+\alpha_{u N}^{V, L R}\right)+N_{c} q_{d}\left(\alpha_{d N}^{V, R R}+\alpha_{d N}^{V, L R}\right)\right. \\
& \left.+q_{e}\left(\alpha_{e N}^{V, R R}+\alpha_{e N}^{V, L R}\right)\right], \\
\dot{\alpha}_{\psi N}^{V, L R}= & \frac{4}{3} e^{2} q_{\psi}\left[N_{c} q_{u}\left(\alpha_{u N}^{V, R R}+\alpha_{u N}^{V, L R}\right)+N_{c} q_{d}\left(\alpha_{d N}^{V, R R}+\alpha_{d N}^{V, L R}\right)\right. \\
& \left.+q_{e}\left(\alpha_{e N}^{V, R R}+\alpha_{e N}^{V, L R}\right)\right],
\end{aligned}
$$

for $\psi=\nu, N, e, u, d$. The non-vanishing electric charges are $q_{e}=-1, q_{u}=2 / 3$ and $q_{d}=-1 / 3$. This automatically implies that $\dot{\alpha}_{N N}^{V, R R}=0$ and $\dot{\alpha}_{\nu N}^{V, L R}=0$; i.e. these operators do not renormalise.

Finally, lepton number violating operators are also induced within our framework when $m_{N} \neq 0$. There are 19 operators violating lepton number by two units and one violating it by four units. We list them in table 4. (The subset of these operators relevant for 


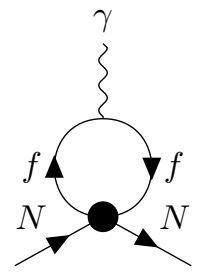

(a)

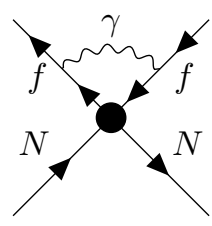

(b)

Figure 11. (a) Renormalisation of the operator $\partial^{\nu} A_{\mu \nu} \bar{N} \gamma^{\mu} N$ by four-fermions. It generates other four-fermions upon using the equations of motion. (b) Self-renormalisation of four-fermions.

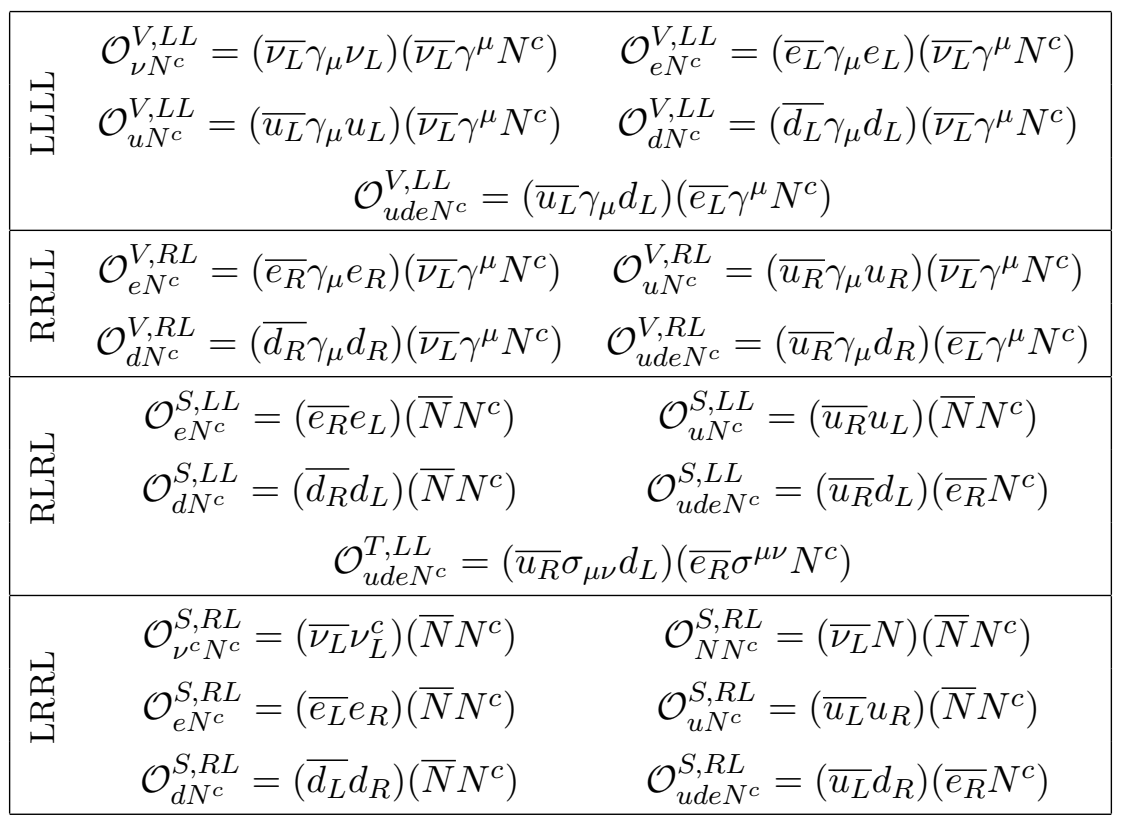

Table 4. List of $\nu$ LEFT lepton number violating operators involving $N^{c}$. The addition of h.c. is implied when needed. Note that the operators obtained from the non-Hermitian operators in the table with multiplication by the imaginary unit are also present.

neutrinoless double beta decay has been recently provided in ref. [67], which also considers higher dimensional operators, the running down to the QCD scale as well as the matching onto the chiral perturbation theory.)

If $m_{N}$ is not vanishing, the $\nu$ SMEFT operators $\mathcal{O}_{N W}$ and $\mathcal{O}_{N B}$ induce, after EWSB, lepton number violating operators proportional to $m_{N} v / \Lambda^{2}$ upon using the equation of motion $i \not \partial N \sim m_{N} N^{c}$. These are: $\mathcal{O}_{\nu N^{c}}^{V, L L}, \mathcal{O}_{e N^{c}}^{V, L L}, \mathcal{O}_{e N^{c}}^{V, R L}, \mathcal{O}_{u N^{c}}^{V, L L}, \mathcal{O}_{u N^{c}}^{V, R L}, \mathcal{O}_{d N^{c}}^{V, L L}$ and $\mathcal{O}_{d N^{c}}^{V, R L}$ ( $Z$ mediated) as well as $\mathcal{O}_{u d e N^{c}}^{V, L L}$ and $\mathcal{O}_{e N^{c}}^{V, L L}(W$ mediated $)$.

Open Access. This article is distributed under the terms of the Creative Commons Attribution License (CC-BY 4.0), which permits any use, distribution and reproduction in any medium, provided the original author(s) and source are credited. 


\section{References}

[1] W. Buchmüller and D. Wyler, Effective Lagrangian Analysis of New Interactions and Flavor Conservation, Nucl. Phys. B 268 (1986) 621 [InSPIRE].

[2] B. Grzadkowski, M. Iskrzynski, M. Misiak and J. Rosiek, Dimension-Six Terms in the Standard Model Lagrangian, JHEP 10 (2010) 085 [arXiv: 1008.4884] [INSPIRE].

[3] I. Brivio and M. Trott, The Standard Model as an Effective Field Theory, Phys. Rept. 793 (2019) 1 [arXiv: 1706.08945] [INSPIRE].

[4] F. del Aguila, S. Bar-Shalom, A. Soni and J. Wudka, Heavy Majorana Neutrinos in the Effective Lagrangian Description: Application to Hadron Colliders, Phys. Lett. B 670 (2009) 399 [arXiv:0806.0876] [INSPIRE].

[5] Y. Liao and X.-D. Ma, Operators up to Dimension Seven in Standard Model Effective Field Theory Extended with Sterile Neutrinos, Phys. Rev. D 96 (2017) 015012 [arXiv: 1612.04527] [INSPIRE].

[6] S. Biondini, N. Brambilla, M.A. Escobedo and A. Vairo, An effective field theory for non-relativistic Majorana neutrinos, JHEP 12 (2013) 028 [arXiv:1307.7680] [INSPIRE].

[7] R. Franceschini et al., Digamma, what next?, JHEP 07 (2016) 150 [arXiv:1604.06446] [INSPIRE].

[8] B. Gripaios and D. Sutherland, An operator basis for the Standard Model with an added scalar singlet, JHEP 08 (2016) 103 [arXiv: 1604.07365] [INSPIRE].

[9] Anisha, S. Das Bakshi, J. Chakrabortty and S. Prakash, Hilbert Series and Plethystics: Paving the path towards 2HDM- and MLRSM-EFT, JHEP 09 (2019) 035 [arXiv: 1905.11047] [INSPIRE].

[10] G. Durieux, F. Maltoni and C. Zhang, Global approach to top-quark flavor-changing interactions, Phys. Rev. D 91 (2015) 074017 [arXiv:1412.7166] [InSPIRE].

[11] M. Chala, J. Santiago and M. Spannowsky, Constraining four-fermion operators using rare top decays, JHEP 04 (2019) 014 [arXiv: 1809.09624] [INSPIRE].

[12] S. Banerjee, M. Chala and M. Spannowsky, Top quark FCNCs in extended Higgs sectors, Eur. Phys. J. C 78 (2018) 683 [arXiv:1806.02836] [InSPIRE].

[13] J. Alcaide, S. Banerjee, M. Chala and A. Titov, Probes of the Standard Model effective field theory extended with a right-handed neutrino, JHEP 08 (2019) 031 [arXiv:1905.11375] [INSPIRE].

[14] A. Caputo, P. Hernández, J. Lopez-Pavon and J. Salvado, The seesaw portal in testable models of neutrino masses, JHEP 06 (2017) 112 [arXiv:1704.08721] [INSPIRE].

[15] J.M. Butterworth, M. Chala, C. Englert, M. Spannowsky and A. Titov, Higgs phenomenology as a probe of sterile neutrinos, Phys. Rev. D 100 (2019) 115019 [arXiv: 1909.04665] [INSPIRE].

[16] I. Bischer and W. Rodejohann, General neutrino interactions from an effective field theory perspective, Nucl. Phys. B 947 (2019) 114746 [arXiv: 1905.08699] [INSPIRE].

[17] E.E. Jenkins, A.V. Manohar and P. Stoffer, Low-Energy Effective Field Theory below the Electroweak Scale: Operators and Matching, JHEP 03 (2018) 016 [arXiv:1709.04486] [INSPIRE]. 
[18] W. Dekens and P. Stoffer, Low-energy effective field theory below the electroweak scale: matching at one loop, JHEP 10 (2019) 197 [arXiv:1908.05295] [INSPIRE].

[19] E.E. Jenkins, A.V. Manohar and M. Trott, Renormalization Group Evolution of the Standard Model Dimension Six Operators I: Formalism and lambda Dependence, JHEP 10 (2013) 087 [arXiv: 1308.2627] [INSPIRE].

[20] E.E. Jenkins, A.V. Manohar and M. Trott, Renormalization Group Evolution of the Standard Model Dimension Six Operators II: Yukawa Dependence, JHEP 01 (2014) 035 [arXiv: 1310.4838$]$ [INSPIRE].

[21] R. Alonso, E.E. Jenkins, A.V. Manohar and M. Trott, Renormalization Group Evolution of the Standard Model Dimension Six Operators III: Gauge Coupling Dependence and Phenomenology, JHEP 04 (2014) 159 [arXiv:1312.2014] [INSPIRE].

[22] E.E. Jenkins, A.V. Manohar and P. Stoffer, Low-Energy Effective Field Theory below the Electroweak Scale: Anomalous Dimensions, JHEP 01 (2018) 084 [arXiv:1711.05270] [INSPIRE].

[23] A. Aparici, K. Kim, A. Santamaria and J. Wudka, Right-handed neutrino magnetic moments, Phys. Rev. D 80 (2009) 013010 [arXiv:0904.3244] [INSPIRE].

[24] S. Bhattacharya and J. Wudka, Dimension-seven operators in the standard model with right handed neutrinos, Phys. Rev. D 94 (2016) 055022 [Erratum ibid. D 95 (2017) 039904] [arXiv: 1505.05264] [INSPIRE].

[25] B. Henning, X. Lu and H. Murayama, How to use the Standard Model effective field theory, JHEP 01 (2016) 023 [arXiv: 1412.1837] [INSPIRE].

[26] B. Henning, X. Lu and H. Murayama, One-loop Matching and Running with Covariant Derivative Expansion, JHEP 01 (2018) 123 [arXiv:1604.01019] [INSPIRE].

[27] S.A.R. Ellis, J. Quevillon, T. You and Z. Zhang, Mixed heavy-light matching in the Universal One-Loop Effective Action, Phys. Lett. B 762 (2016) 166 [arXiv:1604.02445] [INSPIRE].

[28] J. Fuentes-Martin, J. Portoles and P. Ruiz-Femenia, Integrating out heavy particles with functional methods: a simplified framework, JHEP 09 (2016) 156 [arXiv:1607.02142] [INSPIRE].

[29] Z. Zhang, Covariant diagrams for one-loop matching, JHEP 05 (2017) 152 [arXiv: 1610.00710] [INSPIRE].

[30] S.A.R. Ellis, J. Quevillon, T. You and Z. Zhang, Extending the Universal One-Loop Effective Action: Heavy-Light Coefficients, JHEP 08 (2017) 054 [arXiv: 1706. 07765] [INSPIRE].

[31] F. del Aguila, Z. Kunszt and J. Santiago, One-loop effective lagrangians after matching, Eur. Phys. J. C 76 (2016) 244 [arXiv:1602.00126] [InSPIRE].

[32] J.C. Criado, MatchingTools: a Python library for symbolic effective field theory calculations, Comput. Phys. Commun. 227 (2018) 42 [arXiv:1710.06445] [INSPIRE].

[33] S. Das Bakshi, J. Chakrabortty and S.K. Patra, CoDEx: Wilson coefficient calculator connecting SMEFT to UV theory, Eur. Phys. J. C 79 (2019) 21 [arXiv:1808.04403] [INSPIRE].

[34] I. Brivio et al., Computing Tools for the SMEFT, in Computing Tools for the SMEFT, J. Aebischer, M. Fael, A. Lenz, M. Spannowsky and J. Virto eds., 2019, arXiv:1910.11003 [INSPIRE]. 
[35] M. Boggia, R. Gomez-Ambrosio and G. Passarino, Low energy behaviour of standard model extensions, JHEP 05 (2016) 162 [arXiv:1603.03660] [INSPIRE].

[36] M. Jiang, N. Craig, Y.-Y. Li and D. Sutherland, Complete One-Loop Matching for a Singlet Scalar in the Standard Model EFT, JHEP 02 (2019) 031 [arXiv:1811.08878] [INSPIRE].

[37] M.S. Bilenky and A. Santamaria, One loop effective Lagrangian for a standard model with a heavy charged scalar singlet, Nucl. Phys. B 420 (1994) 47 [hep-ph/9310302] [INSPIRE].

[38] K. Kannike, M. Raidal, D.M. Straub and A. Strumia, Anthropic solution to the magnetic muon anomaly: the charged see-saw, JHEP 02 (2012) 106 [Erratum ibid. 1210 (2012) 136] [arXiv:1111.2551] [INSPIRE].

[39] R. Dermisek and A. Raval, Explanation of the Muon g-2 Anomaly with Vectorlike Leptons and its Implications for Higgs Decays, Phys. Rev. D 88 (2013) 013017 [arXiv:1305.3522] [INSPIRE].

[40] A.S. De Jesus, S. Kovalenko, F.S. Queiroz, C. Siqueira and K. Sinha, Vector-Like Leptons and Inert Scalar Triplet: Lepton Flavor Violation, $g-2$ and Collider Searches, arXiv: 2004.01200 [INSPIRE].

[41] G.C. Joshi and R.R. Volkas, Extended weak isospin and fermion masses in a unified model, Phys. Rev. D 45 (1992) 1711 [InSPIRE].

[42] D. Guadagnoli, R.N. Mohapatra and I. Sung, Gauged Flavor Group with Left-Right Symmetry, JHEP 04 (2011) 093 [arXiv: 1103.4170] [INSPIRE].

[43] R.N. Mohapatra and Y. Zhang, TeV Scale Universal Seesaw, Vacuum Stability and Heavy Higgs, JHEP 06 (2014) 072 [arXiv:1401.6701] [INSPIRE].

[44] R.M. Fonseca, On the chirality of the SM and the fermion content of GUTs, Nucl. Phys. B 897 (2015) 757 [arXiv:1504.03695] [INSPIRE].

[45] M. Chala, $h \rightarrow \gamma \gamma$ excess and Dark Matter from Composite Higgs Models, JHEP 01 (2013) 122 [arXiv: 1210.6208] [INSPIRE].

[46] G. Cacciapaglia, T. Ma, S. Vatani and Y. Wu, Towards a fundamental safe theory of composite Higgs and Dark Matter, arXiv:1812.04005 [INSPIRE].

[47] D.B. Kaplan, Flavor at SSC energies: A New mechanism for dynamically generated fermion masses, Nucl. Phys. B 365 (1991) 259 [InSPIRE].

[48] A.V. Manohar, Introduction to Effective Field Theories, in Les Houches summer school: EFT in Particle Physics and Cosmology, Les Houches France (2017) [arXiv:1804.05863] [INSPIRE].

[49] J.C. Criado, BasisGen: automatic generation of operator bases, Eur. Phys. J. C 79 (2019) 256 [arXiv: 1901.03501] [INSPIRE].

[50] R.M. Fonseca, Enumerating the operators of an effective field theory, Phys. Rev. D 101 (2020) 035040 [arXiv:1907.12584] [INSPIRE].

[51] W.S. Deans and J.A. Dixon, Theory of Gauge Invariant Operators: Their Renormalization and S Matrix Elements, Phys. Rev. D 18 (1978) 1113 [INSPIRE].

[52] H.D. Politzer, Power Corrections at Short Distances, Nucl. Phys. B 172 (1980) 349 [INSPIRE].

[53] J.C. Criado and M. Pérez-Victoria, Field redefinitions in effective theories at higher orders, JHEP 03 (2019) 038 [arXiv: 1811.09413] [INSPIRE]. 
[54] J. Ellis, TikZ-Feynman: Feynman diagrams with TikZ, Comput. Phys. Commun. 210 (2017) 103 [arXiv: 1601.05437 ] [INSPIRE].

[55] Particle Data Group collaboration, Review of Particle Physics, Phys. Rev. D 98 (2018) 030001 [INSPIRE].

[56] L. Duarte, J. Peressutti and O.A. Sampayo, Majorana neutrino decay in an Effective Approach, Phys. Rev. D 92 (2015) 093002 [arXiv:1508.01588] [INSPIRE].

[57] L3 collaboration, Search for new physics in energetic single photon production in $e^{+} e^{-}$ annihilation at the $Z$ resonance, Phys. Lett. B 412 (1997) 201 [INSPIRE].

[58] CMS collaboration, Search for new physics in final states with a single photon and missing transverse momentum in proton-proton collisions at $\sqrt{s}=13 \mathrm{TeV}$, JHEP 02 (2019) 074 [arXiv: 1810.00196] [INSPIRE].

[59] J.M. Butterworth, D. Grellscheid, M. Krämer, B. Sarrazin and D. Yallup, Constraining new physics with collider measurements of Standard Model signatures, JHEP 03 (2017) 078 [arXiv: 1606.05296] [INSPIRE].

[60] L. Duarte, J. Peressutti and O.A. Sampayo, Not-that-heavy Majorana neutrino signals at the LHC, J. Phys. G 45 (2018) 025001 [arXiv:1610.03894] [INSPIRE].

[61] B.C. Canas, O.G. Miranda, A. Parada, M. Tortola and J.W.F. Valle, Updating neutrino magnetic moment constraints, Phys. Lett. B 753 (2016) 191 [arXiv:1510. 01684] [INSPIRE].

[62] M. Redi, Leptons in Composite MFV, JHEP 09 (2013) 060 [arXiv:1306.1525] [INSPIRE].

[63] Q.-H. Cao, G. Li, K.-P. Xie and J. Zhang, Searching for Weak Singlet Charged Scalar at the Large Hadron Collider, Phys. Rev. D 97 (2018) 115036 [arXiv:1711.02113] [INSPIRE].

[64] ATLAS collaboration, Search for electroweak production of supersymmetric particles in final states with two or three leptons at $\sqrt{s}=13 \mathrm{TeV}$ with the ATLAS detector, Eur. Phys. J. C 78 (2018) 995 [arXiv: 1803. 02762] [INSPIRE].

[65] J. Alcaide and N.I. Mileo, LHC sensitivity to singly-charged scalars decaying into electrons and muons, arXiv:1906.08685 [INSPIRE].

[66] J.L. Aalberts et al., Precision constraints on radiative neutrino decay with CMB spectral distortion, Phys. Rev. D 98 (2018) 023001 [arXiv: 1803.00588] [INSPIRE].

[67] W. Dekens, J. de Vries, K. Fuyuto, E. Mereghetti and G. Zhou, Sterile neutrinos and neutrinoless double beta decay in effective field theory, arXiv:2002.07182 [INSPIRE]. 